\title{
OLANZAPINE SALTS AND DIVERSITY IN MOLECULAR PACKING
}

Kashyap Kumar Sarmah, ${ }^{\mathrm{a}}$ Ayushree Sarma, ${ }^{\mathrm{a}}$ Karabi Roy, ${ }^{\mathrm{a}}$ Dharmaraj R. Rao, ${ }^{\mathrm{b}}$ Ranjit Thakuria ${ }^{\mathrm{a}, *}$

${ }^{a}$ Department of Chemistry, Gauhati University, Guwahati 781014, Assam, India

${ }^{\mathrm{b}}$ Cipla Ltd., Research \& Development Centre, L B S Marg, Vikhroli, Mumbai 400083

ORTEP diagrams of the OLN salt structures:

1. OLN•Gly salt (1:1):

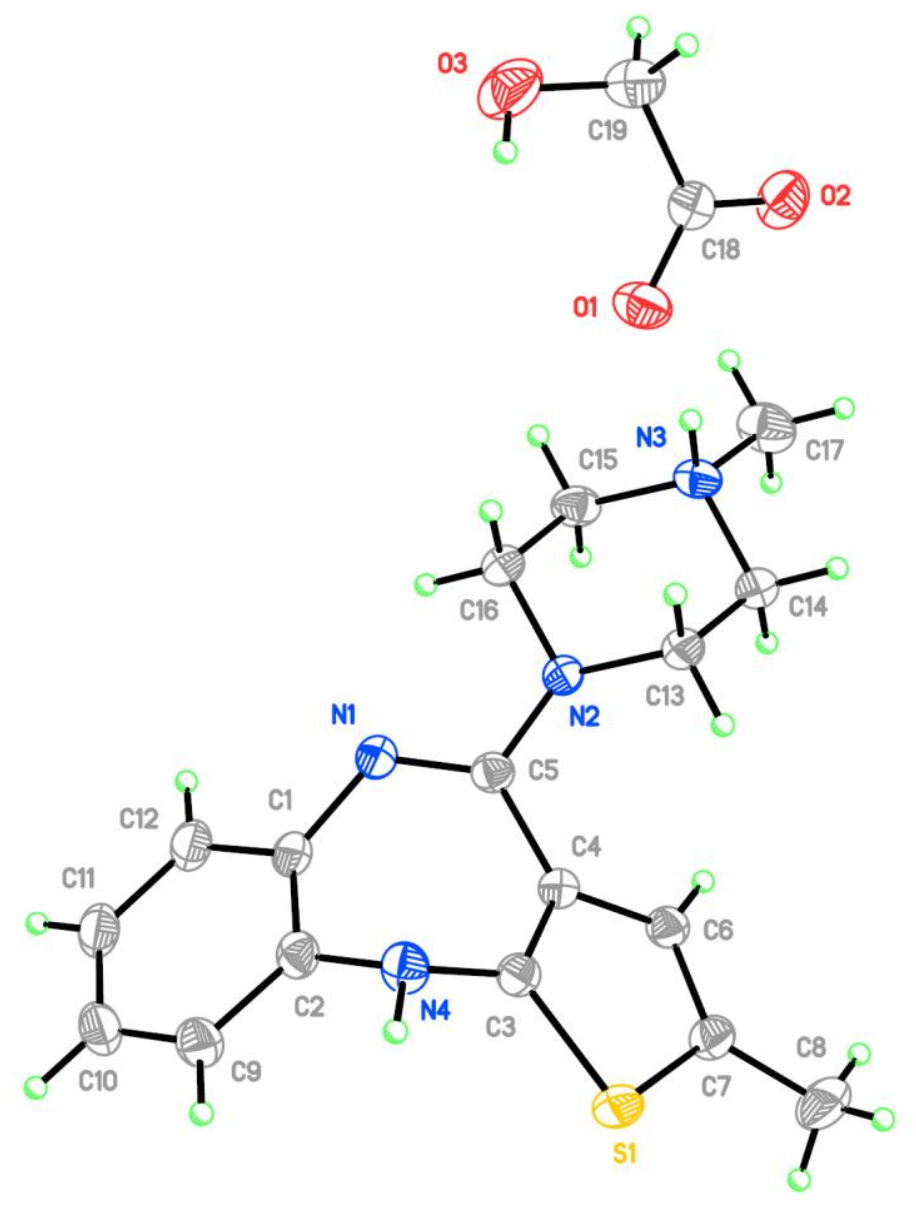

Figure S1: ORTEP diagram of OLN•Gly salt with $35 \%$ probability ellipsoid.

2. OLN•pTSA•H $\mathrm{H}_{2} \mathrm{O}$ salt hydrate (1:1:1): 


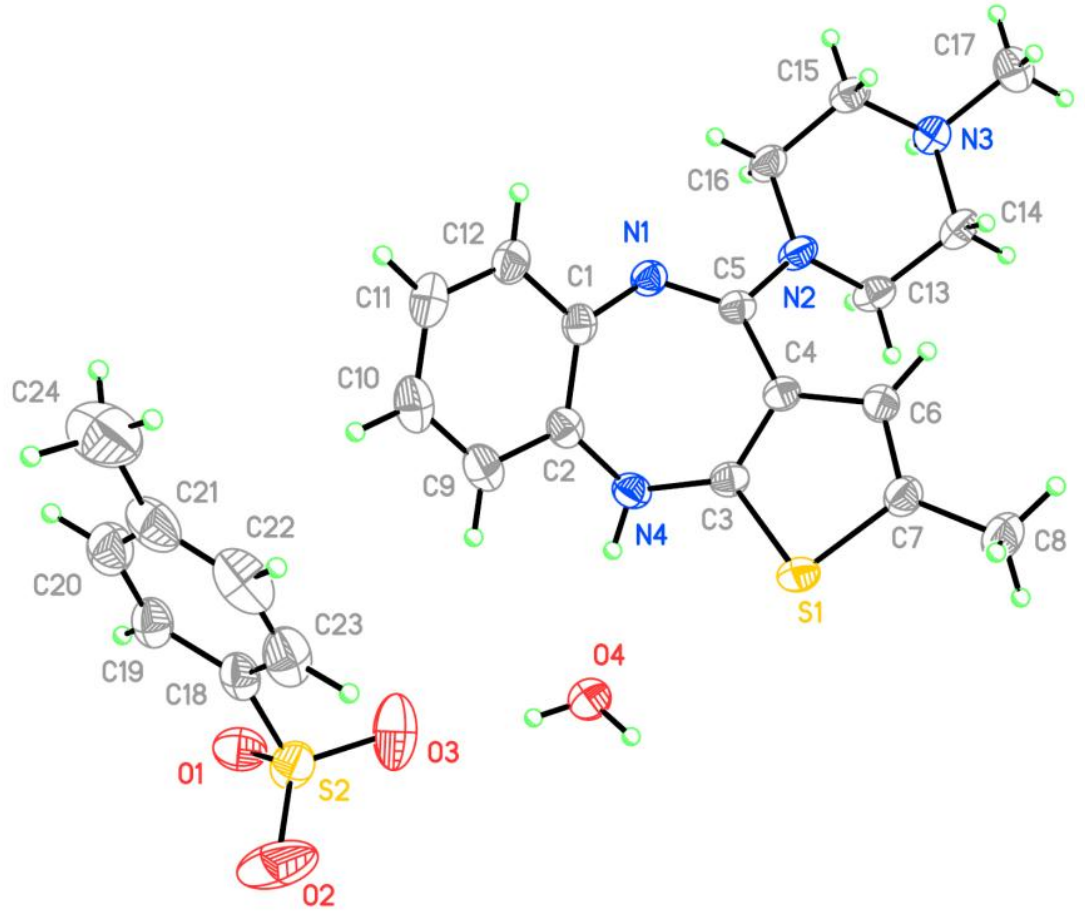

Figure S2: ORTEP diagram of ONL $\cdot p T S A \cdot \mathrm{H}_{2} \mathrm{O}$ crystal structure with $35 \%$ probability ellipsoid.

3. $\mathrm{OLN} \cdot 4 \mathrm{HBA} \cdot \mathrm{CH}_{3} \mathrm{CN}$ salt solvate $(2: 2: 1)$ : 

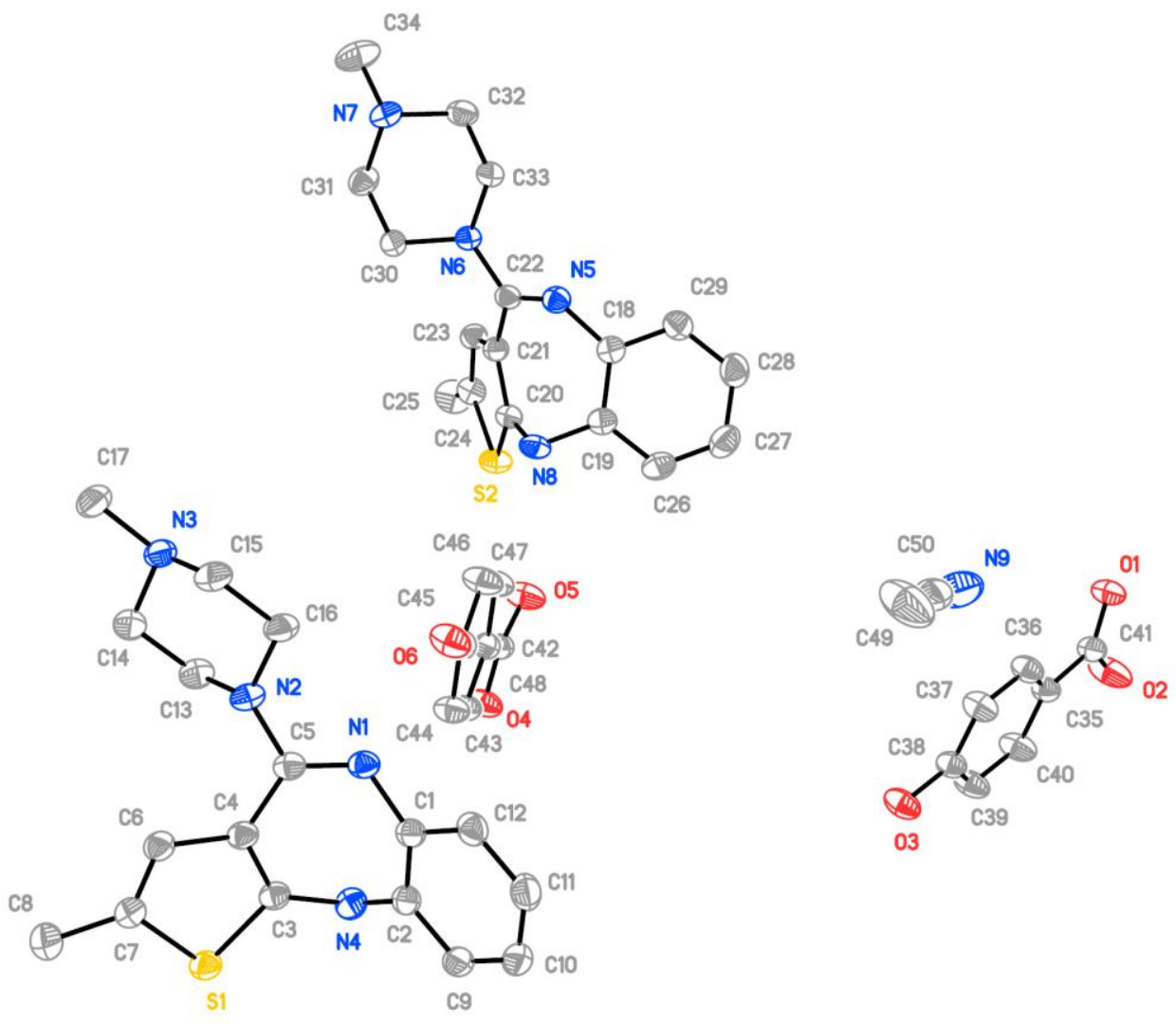

Figure S3: ORTEP diagram of OLN $\bullet 4 \mathrm{HBA} \cdot \mathrm{CH}_{3} \mathrm{CN}$ salt solvate crystal structure with $35 \%$ probability ellipsoid. $\mathrm{H}$ atoms are omitted for clarity.

\section{OLN•24DHBA salt (1:1):}




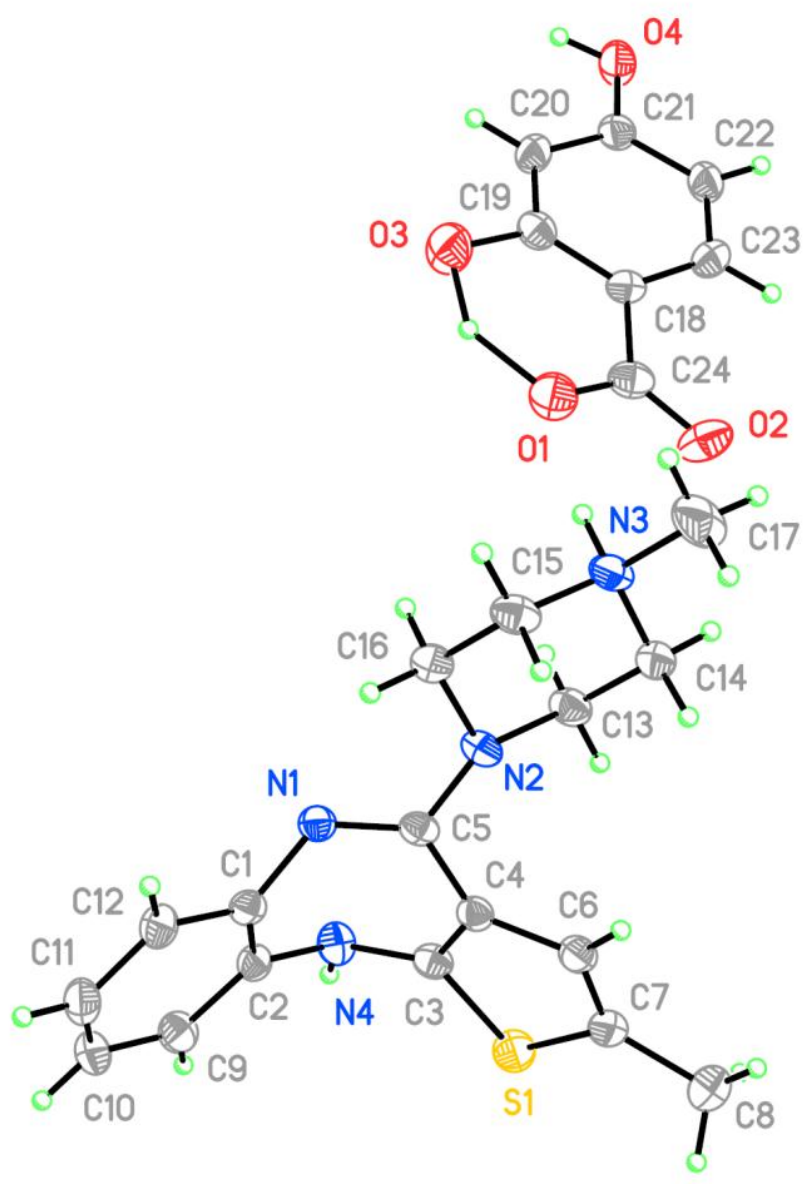

Figure S4: ORTEP diagram of OLN•24DHBA salt crystal structure with $35 \%$ probability ellipsoid.

\section{OLN•25DHBA salt (1:1):}




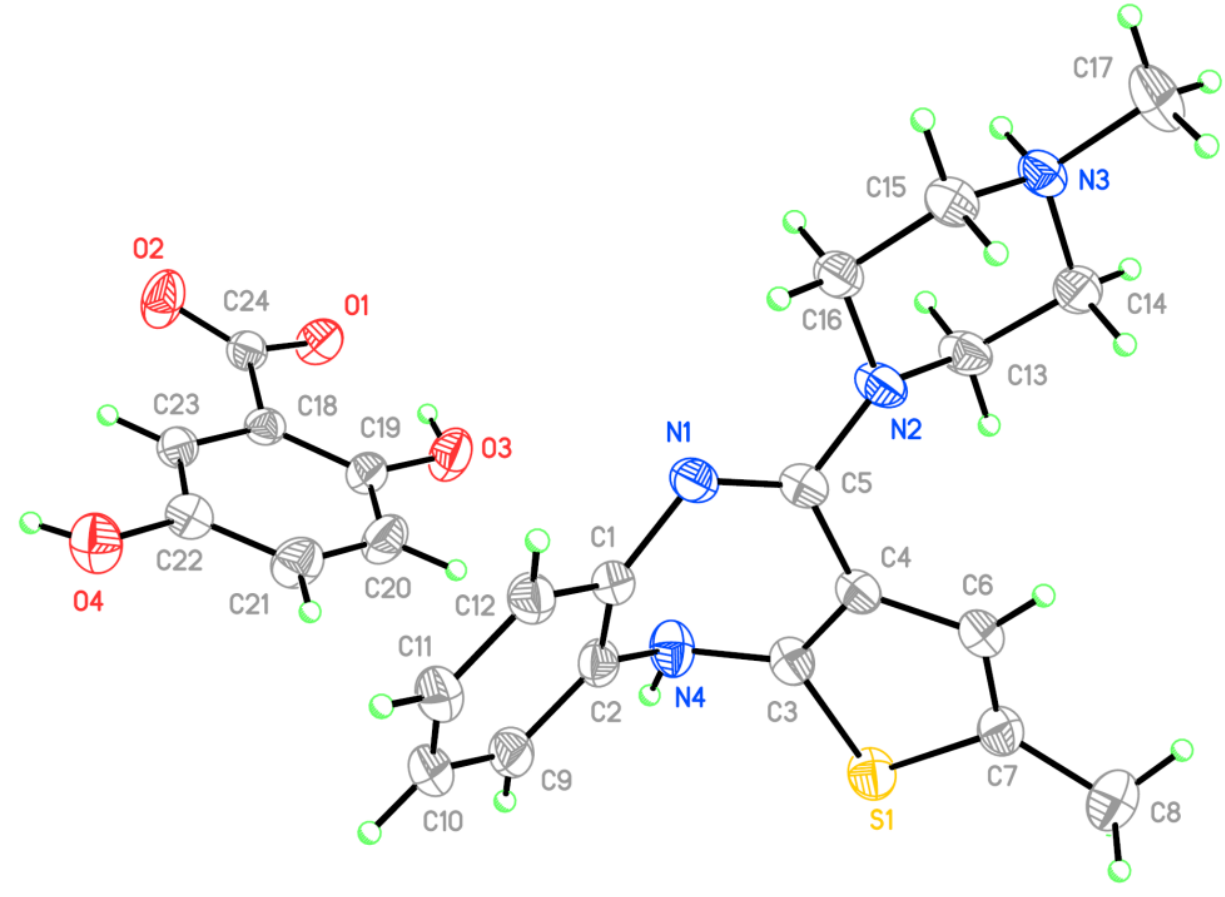

Figure S5: ORTEP diagram of OLN•25DHBA salt structure with $35 \%$ probability ellipsoid.

\section{OLN•26DHBA salt $(1: 1)$ :}

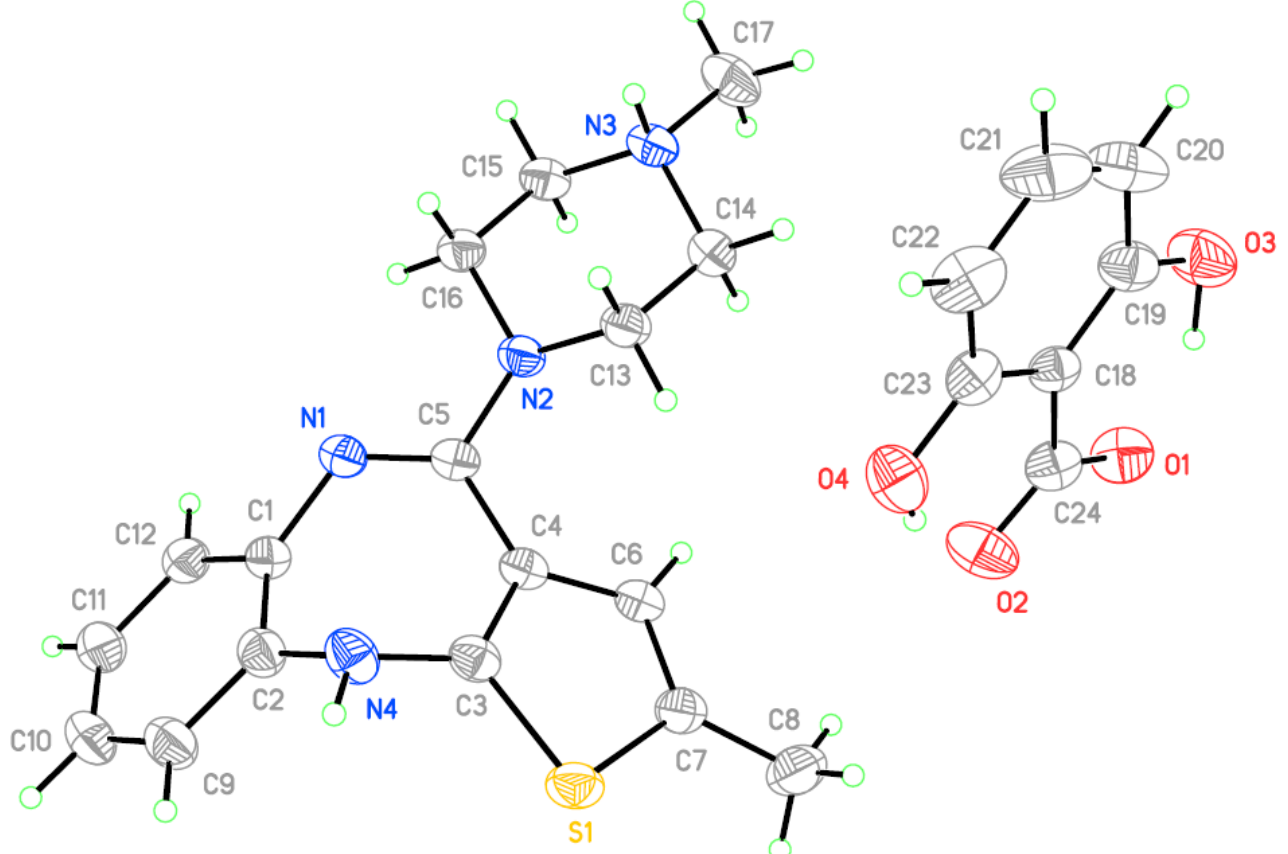

Figure S6: ORTEP diagram of OLN•26DHBA salt structure with $35 \%$ probability ellipsoid. 
Table S1. Hydrogen bonds in the crystal structures of OLN salts

\begin{tabular}{|c|c|c|c|c|c|}
\hline Compound & Interaction & 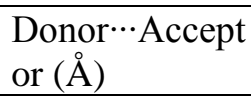 & 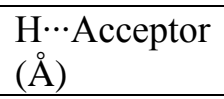 & $\begin{array}{l}\text { Donor- } \\
H^{*} \cdots \text { Acceptor }\left({ }^{\circ}\right)\end{array}$ & Symmetry code \\
\hline \multirow[t]{5}{*}{$\mathrm{OLN} \cdot \mathrm{Gly}$} & $\mathrm{N} 3^{+}-\mathrm{H} 3 \mathrm{~A} \cdots \mathrm{O} 2$ & $3.322(2)$ & 2.579 & 129 & $\mathrm{x}, \mathrm{y}, \mathrm{z}$ \\
\hline & $\mathrm{N} 3^{+}-\mathrm{H} 3 \mathrm{~A} \cdots \mathrm{O}^{-}$ & $2.703(2)$ & 1.690 & 167 & $\mathrm{x}, \mathrm{y}, \mathrm{z}$ \\
\hline & $\mathrm{O} 3-\mathrm{H} 3 \mathrm{~B} \cdots \mathrm{O}^{-}$ & $2.747(2)$ & 2.489 & 96 & $\mathrm{x}, \mathrm{y}, \mathrm{z}$ \\
\hline & $\mathrm{N} 4-\mathrm{H} 4 \mathrm{~A} \cdots \mathrm{O} 2$ & $2.893(2)$ & 1.866 & 175 & $\mathrm{x},+\mathrm{y}-1, \mathrm{z}+1$ \\
\hline & $\mathrm{O} 3-\mathrm{H} 3 \mathrm{~B} \cdots \mathrm{O} 1^{-}$ & $2.792(2)$ & 1.925 & 153 & $-\mathrm{x}+1,-\mathrm{y}+1,-\mathrm{z}+1$ \\
\hline \multirow{9}{*}{$\begin{array}{l}\mathrm{OLN} \bullet \mathrm{pTSA} \bullet \\
\mathrm{H}_{2} \mathrm{O}\end{array}$} & $\mathrm{N} 4-\mathrm{H} 4 \mathrm{~A} \cdots \mathrm{S} 1$ & $2.743(2)$ & 2.784 & 77 & $\mathrm{x}, \mathrm{y}, \mathrm{z}$ \\
\hline & $\mathrm{N}^{+}-\mathrm{H} 3 \mathrm{~A}^{\cdots} \cdots \mathrm{S} 2$ & $3.548(.001)$ & 2.620 & 150 & $\begin{array}{l}\mathrm{x}-1 / 2,-\mathrm{y}+1 / 2,- \\
\mathrm{z}+1\end{array}$ \\
\hline & $\mathrm{N} 4-\mathrm{H} 4 \mathrm{~A} \cdots \mathrm{O} 4$ & $2.920(2)$ & 1.901 & 169 & $\mathrm{x}, \mathrm{y}, \mathrm{z}$ \\
\hline & C16-H16A $\cdots \mathrm{N} 1$ & $2.756(.002)$ & 2.371 & 99 & $\mathrm{x}, \mathrm{y}, \mathrm{z}$ \\
\hline & 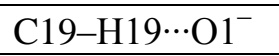 & $2.893(3)$ & 2.485 & 101 & $\mathrm{x}, \mathrm{y}, \mathrm{z}$ \\
\hline & $\mathrm{O} 4-\mathrm{H} 4 \mathrm{~B} \cdots \mathrm{O} 3$ & $2.761(3)$ & 1.838 & 167 & $\mathrm{x}, \mathrm{y}, \mathrm{z}$ \\
\hline & $\mathrm{N} 3^{+}-\mathrm{H} 3 \mathrm{~A}^{\cdots} \cdots \mathrm{O}^{-}$ & $2.742(2)$ & 1.729 & 167 & $\begin{array}{l}\mathrm{x}-1 / 2,-\mathrm{y}+1 / 2,- \\
\mathrm{z}+1\end{array}$ \\
\hline & $\mathrm{N} 3{ }^{+}-\mathrm{H} 3 \mathrm{~A} \cdots \mathrm{O} 3$ & $3.345(3)$ & 2.649 & 125 & $\begin{array}{l}\mathrm{x}-1 / 2,-\mathrm{y}+1 / 2,- \\
\mathrm{z}+1\end{array}$ \\
\hline & $\mathrm{O} 4-\mathrm{H} 4 \mathrm{C} \cdots \mathrm{N} 1$ & $2.954(2)$ & 2.017 & 177 & $\begin{array}{l}-\mathrm{x}+1 / 2, \quad+\mathrm{y}+1 / 2, \\
+\mathrm{z}\end{array}$ \\
\hline \multirow{9}{*}{$\begin{array}{l}\mathrm{OLN} \bullet 4 \mathrm{HBA} \\
\cdot \mathrm{CH}_{3} \mathrm{CN}\end{array}$} & O6-H6A $\cdots \mathrm{N} 1$ & $2.729(2)$ & 1.792 & 178 & $\mathrm{x}, \mathrm{y}, \mathrm{z}$ \\
\hline & N8-H8A $\cdots \mathrm{O} 5$ & $2.891(2)$ & 1.863 & 175 & $\mathrm{x}, \mathrm{y}, \mathrm{z}$ \\
\hline & $\mathrm{N} 4-\mathrm{H} 4 \mathrm{~A} \cdots \mathrm{S} 1$ & $2.724(2)$ & 2.762 & 77 & $\mathrm{x}, \mathrm{y}, \mathrm{z}$ \\
\hline & $\mathrm{N} 4-\mathrm{H} 4 \mathrm{~A} \cdots \mathrm{O} 2$ & $2.824(3)$ & 1.803 & 170 & $-\mathrm{x}+1,-\mathrm{y}+2,-\mathrm{z}+1$ \\
\hline & $\mathrm{N} 3^{+}-\mathrm{H} 3 \mathrm{~A} \cdots \mathrm{O} 1^{-}$ & $2.626(2)$ & 1.601 & 173 & $-\mathrm{x}+1,-\mathrm{y}+1,-\mathrm{z}+1$ \\
\hline & $\mathrm{N} 3^{+}-\mathrm{H} 3 \mathrm{~A} \cdots \mathrm{O} 2$ & $3.183(2)$ & 2.594 & 116 & $-\mathrm{x}+1,-\mathrm{y}+1,-\mathrm{z}+1$ \\
\hline & $\mathrm{O} 3-\mathrm{H} 3 \mathrm{~B} \cdots \mathrm{N} 5$ & $2.780(2)$ & 1.872 & 162 & $-\mathrm{x}+1,-\mathrm{y}+1,-\mathrm{z}+1$ \\
\hline & $\mathrm{N}^{+}-\mathrm{H} 7 \mathrm{~A} \cdots \mathrm{O} 4^{-}$ & $2.563(2)$ & 1.533 & 179 & $\mathrm{x},+\mathrm{y}-1,+\mathrm{z}$ \\
\hline & $\mathrm{N}^{+}-\mathrm{H} 7 \mathrm{~A} \cdots \mathrm{O} 5$ & $3.296(2)$ & 2.628 & 122 & $\mathrm{x},+\mathrm{y}-1,+\mathrm{z}$ \\
\hline \multirow{7}{*}{$\begin{array}{l}\mathrm{OLN} \cdot 24 \mathrm{DH} \\
\mathrm{BA}\end{array}$} & $\mathrm{N} 3^{+}-\mathrm{H} 3 \mathrm{~A} \cdots \mathrm{O}^{-}$ & $2.701(3)$ & 1.703 & 162 & $\mathrm{x}, \mathrm{y}, \mathrm{z}$ \\
\hline & $\mathrm{N} 3^{+}-\mathrm{H} 3 \mathrm{~A} \cdots \mathrm{O} 2$ & $3.068(3)$ & 2.449 & 118 & $\mathrm{x}, \mathrm{y}, \mathrm{z}$ \\
\hline & N4-H4A $\cdots$ S1 & $2.751(2)$ & 2.758 & 79 & $\mathrm{x}, \mathrm{y}, \mathrm{z}$ \\
\hline & $\mathrm{O}^{-}-\mathrm{H} 3 \mathrm{~B} \cdots \mathrm{O} 3$ & $2.572(3)$ & 1.706 & 152 & $\mathrm{x}, \mathrm{y}, \mathrm{z}$ \\
\hline & $\mathrm{O} 3-\mathrm{H} 3 \mathrm{~B} \cdots \mathrm{O} 1^{-}$ & $2.572(3)$ & 1.723 & 149 & $\mathrm{x}, \mathrm{y}, \mathrm{z}$ \\
\hline & $\mathrm{N} 4-\mathrm{H} 4 \mathrm{~A} \cdots \mathrm{O} 2$ & $2.999(3)$ & 1.977 & 171 & $-\mathrm{x}+1,-\mathrm{y}+1,-\mathrm{z}+1$ \\
\hline & $\mathrm{O} 4-\mathrm{H} 4 \mathrm{~B} \cdots \mathrm{N} 1$ & $2.749(3)$ & 1.824 & 169 & $\begin{array}{l}-\mathrm{x}+1 / 2+1, \quad+\mathrm{y}- \\
1 / 2,-\mathrm{z}+1 / 2+1\end{array}$ \\
\hline \multirow{5}{*}{$\begin{array}{l}\mathrm{OLN} \cdot 25 \mathrm{DH} \\
\mathrm{BA}\end{array}$} & O3-H3B $\cdots{ }^{-} 1^{-}$ & $2.581(2)$ & 1.732 & 149 & $\mathrm{x}, \mathrm{y}, \mathrm{z}$ \\
\hline & $\mathrm{N} 3^{+}-\mathrm{H} 3 \mathrm{~A} \cdots \mathrm{O} 1^{-}$ & $2.691(2)$ & 1.663 & 176 & $-\mathrm{x}+1,-\mathrm{y}+1,-\mathrm{z}+1$ \\
\hline & $\mathrm{N} 3^{+}-\mathrm{H} 3 \mathrm{~A} \cdots \mathrm{O} 2$ & $3.254(2)$ & 2.624 & 119 & $-\mathrm{x}+1,-\mathrm{y}+1,-\mathrm{z}+1$ \\
\hline & $\mathrm{N} 4-\mathrm{H} 4 \mathrm{~A} \cdots \mathrm{O} 2$ & $3.478(2)$ & 2.451 & 175 & $\mathrm{x}+1,+\mathrm{y},+\mathrm{z}$ \\
\hline & $\mathrm{O} 4-\mathrm{H} 4 \mathrm{~B} \cdots \mathrm{O} 2$ & $2.737(2)$ & 1.801 & 175 & $-\mathrm{x},-\mathrm{y}+1,-\mathrm{z}+2$ \\
\hline
\end{tabular}




\begin{tabular}{|c|c|c|c|c|c|}
\hline \multirow{5}{*}{$\begin{array}{l}\mathrm{OLN} \cdot 26 \mathrm{DH} \\
\mathrm{BA}\end{array}$} & $\mathrm{O} 3-\mathrm{H} 3 \mathrm{~B} \cdots \mathrm{O} 1$ & $2.554(2)$ & 1.674 & 155 & $\mathrm{x}, \mathrm{y}, \mathrm{z}$ \\
\hline & $\mathrm{O} 4-\mathrm{H} 4 \mathrm{~B} \cdots \mathrm{O} 2$ & $2.491(2)$ & 1.595 & 158 & $\mathrm{x}, \mathrm{y}, \mathrm{z}$ \\
\hline & N4-H4A $\cdots \mathrm{O} 4$ & $3.051(2)$ & 2.031 & 170 & $-\mathrm{x}+1,-\mathrm{y}+1,-\mathrm{z}+2$ \\
\hline & $\mathrm{N} 3-\mathrm{H} 3 \mathrm{~A} \cdots \mathrm{O} 1$ & $2.734(2)$ & 1.772 & 154 & $\mathrm{x},+\mathrm{y}-1,+\mathrm{z}$ \\
\hline & $\mathrm{N} 3-\mathrm{H} 3 \mathrm{~A} \cdots \mathrm{O} 2$ & $3.542(2)$ & 2.666 & 143 & $\mathrm{x},+\mathrm{y}-1,+\mathrm{z}$ \\
\hline
\end{tabular}

\section{Powder X-ray Diffraction (PXRD) analysis}

Powder X-ray Diffraction analysis of all the olanzapinium salts were performed on powder material prepared using liquid assisted grinding (LAG) in presence of few drops of acetonitrile as liquid. Olanzapine with 34DHBA and 35DHBA resulted in multi-component system, characterized using FT-IR, DSC and TGA. However, suitable single crystal could not be generated to get more information about hydrogen bond as well as crystal packing. The PXRD of all the olanzapinium salts matches well with the calculated powder pattern of the corresponding single crystal data (Figure S7); except OLN•4HBA salt as the crystal structure contains acetonitrile solvent molecule in the asymmetric unit.

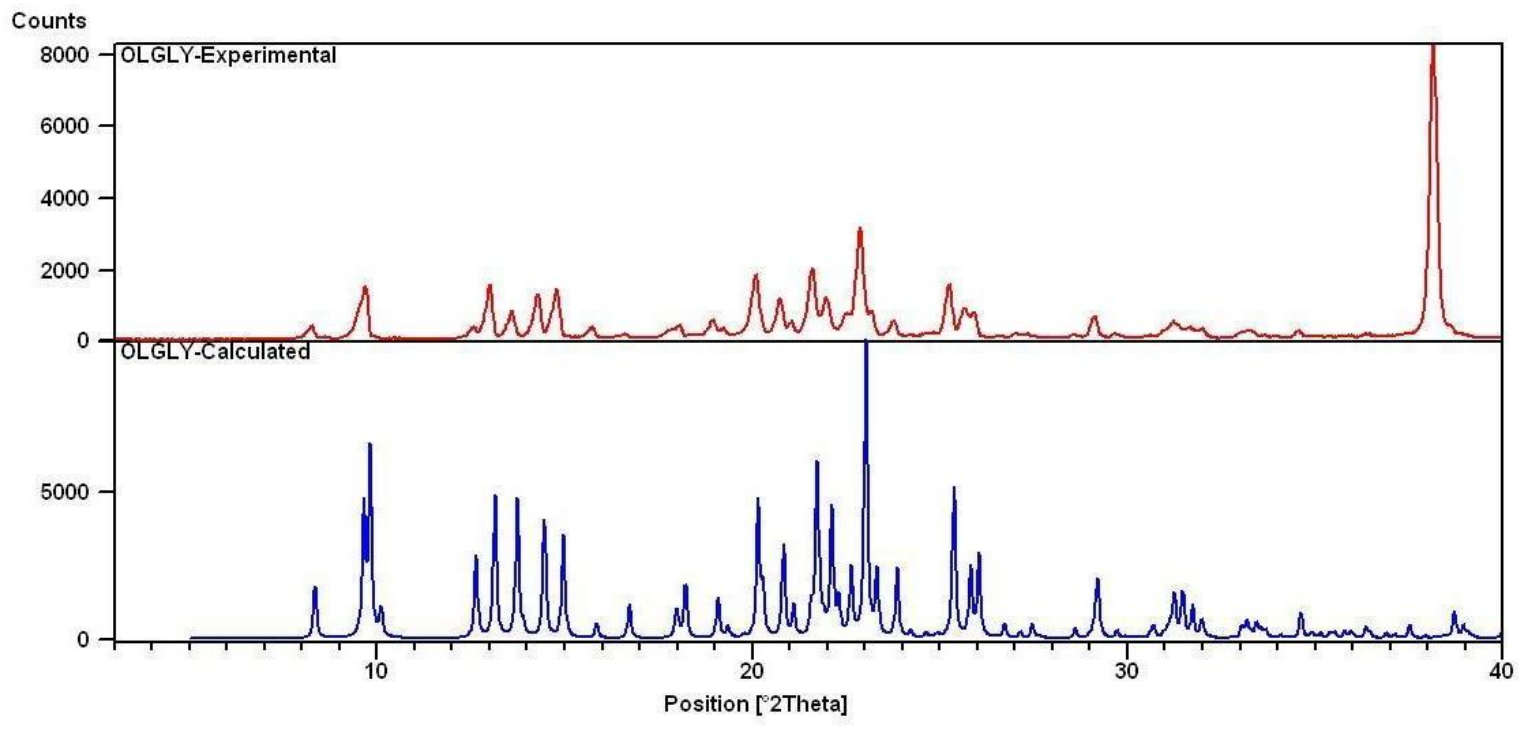

(a) Experimental (red) vs. calculated (blue) PXRD pattern of OLN•Gly salt. 


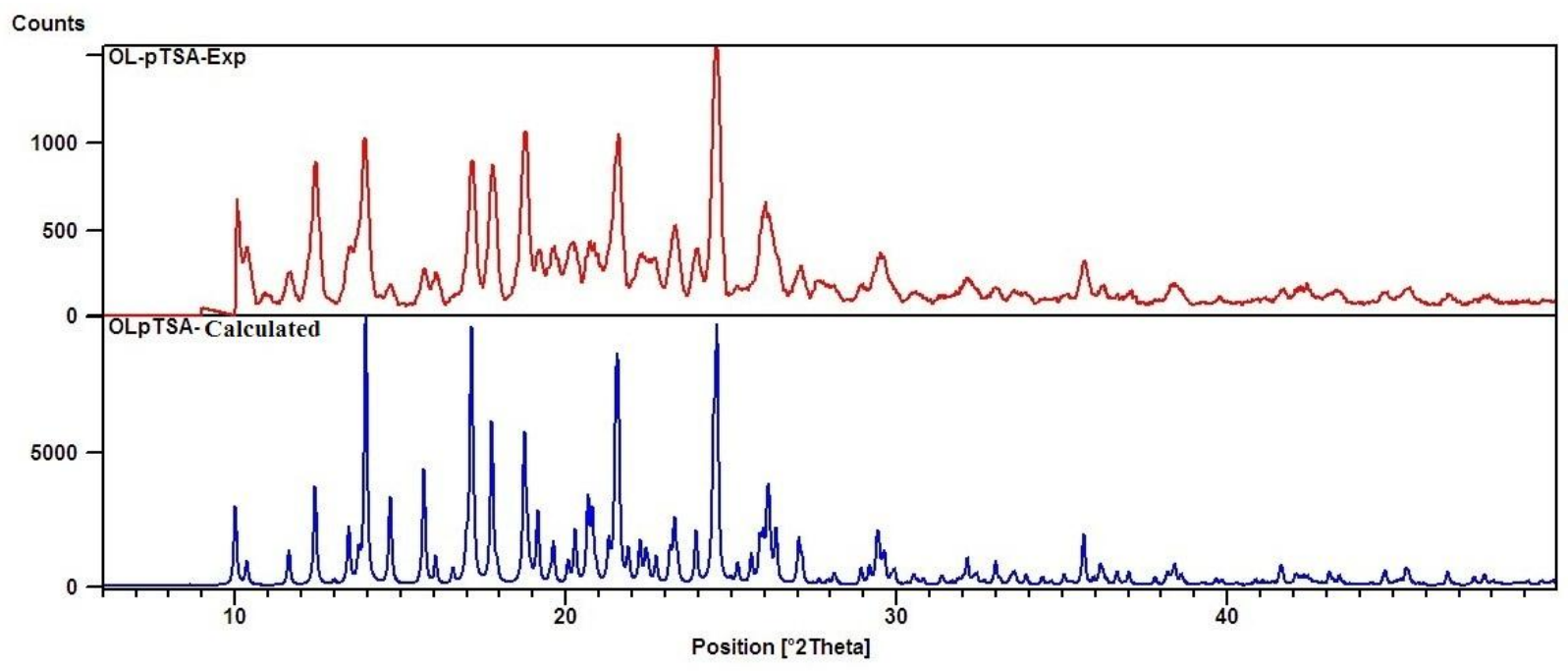

(b) Experimental (red) vs. calculated (blue) PXRD pattern of OLN•pTSA salt.

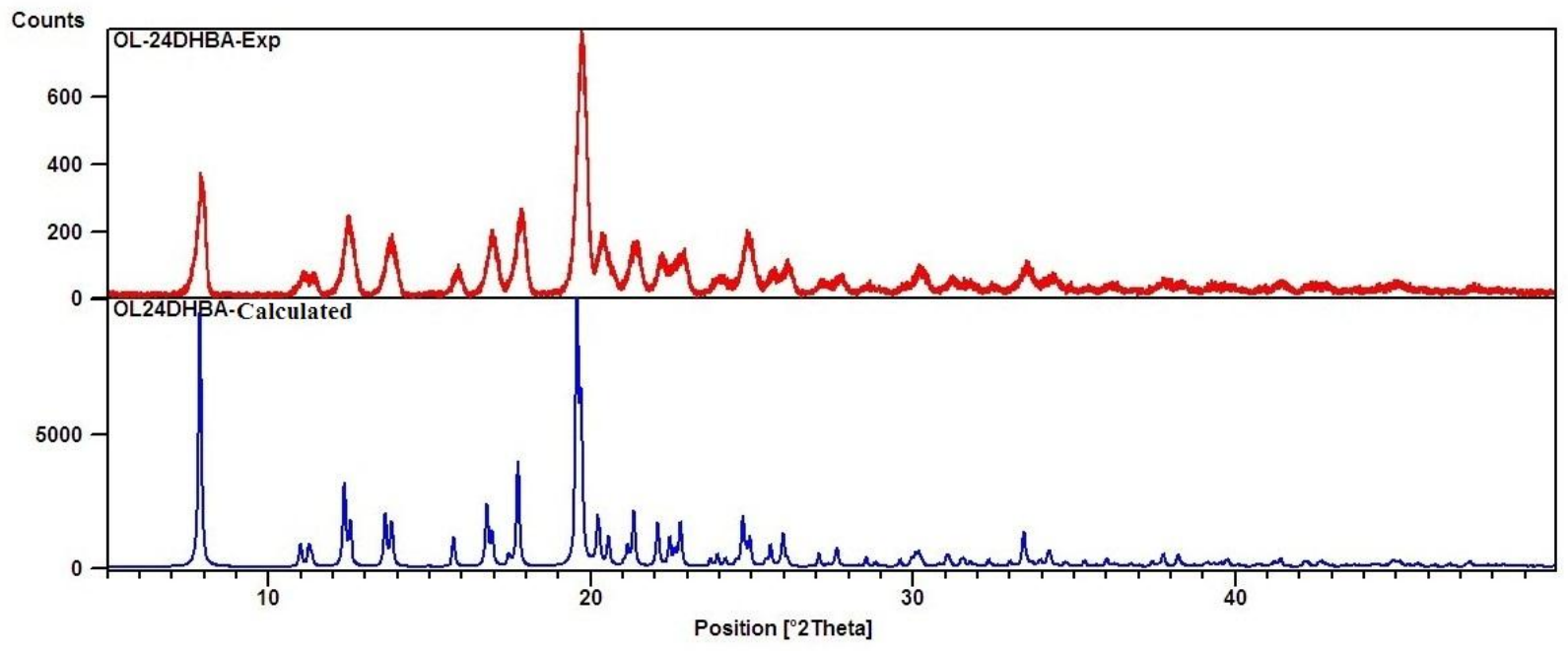

(c) Experimental (red) vs. calculated (blue) PXRD pattern of OLN•24DHBA salt. 


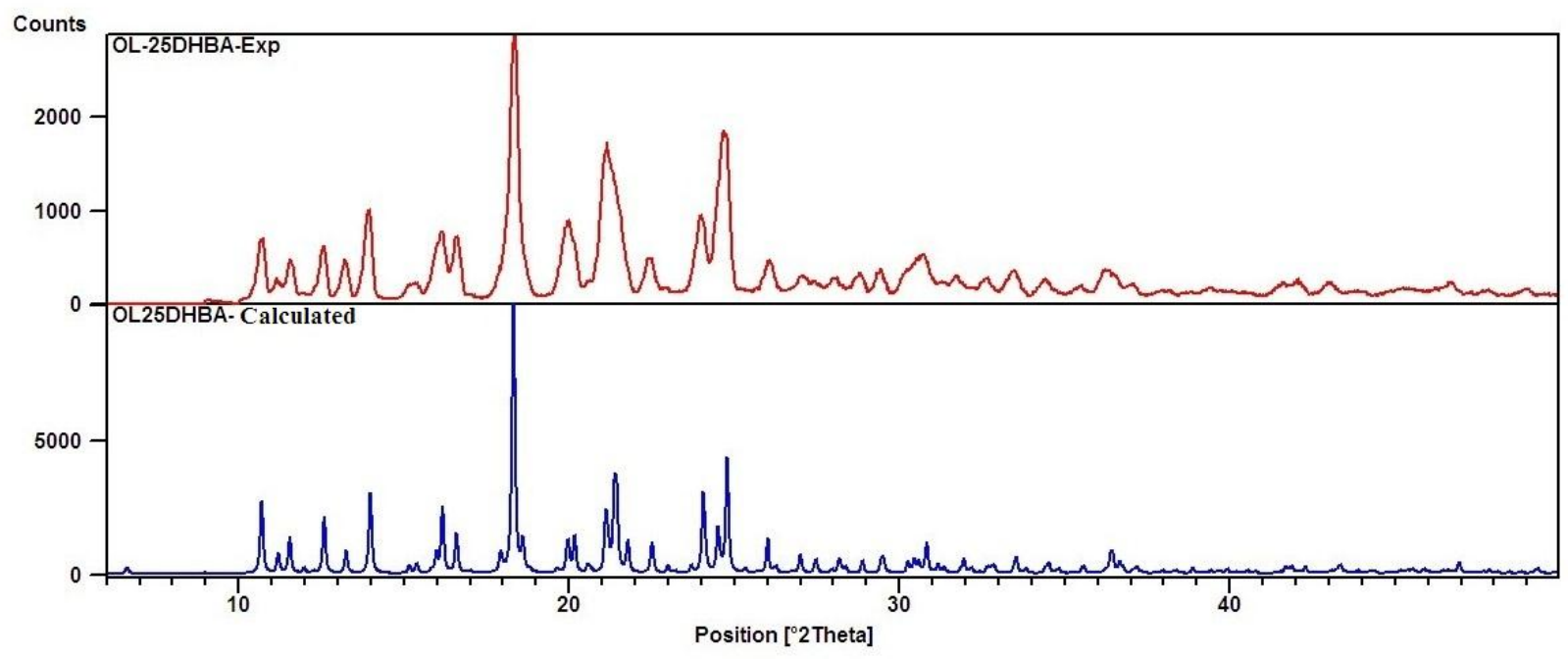

(d) Experimental (red) vs. calculated (blue) PXRD pattern of OLN•25DHBA salt.

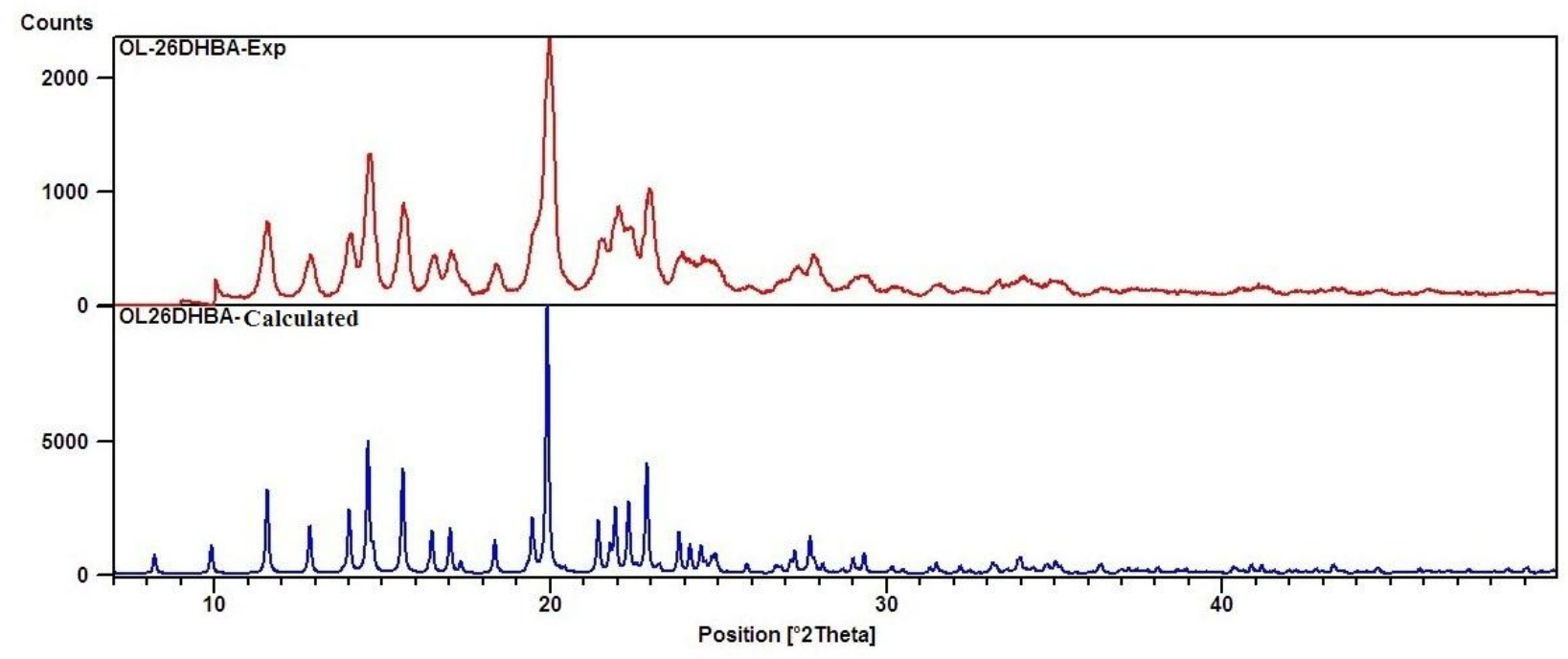

(e) Experimental (red) vs. calculated (blue) PXRD pattern of OLN•26DHBA salt. 


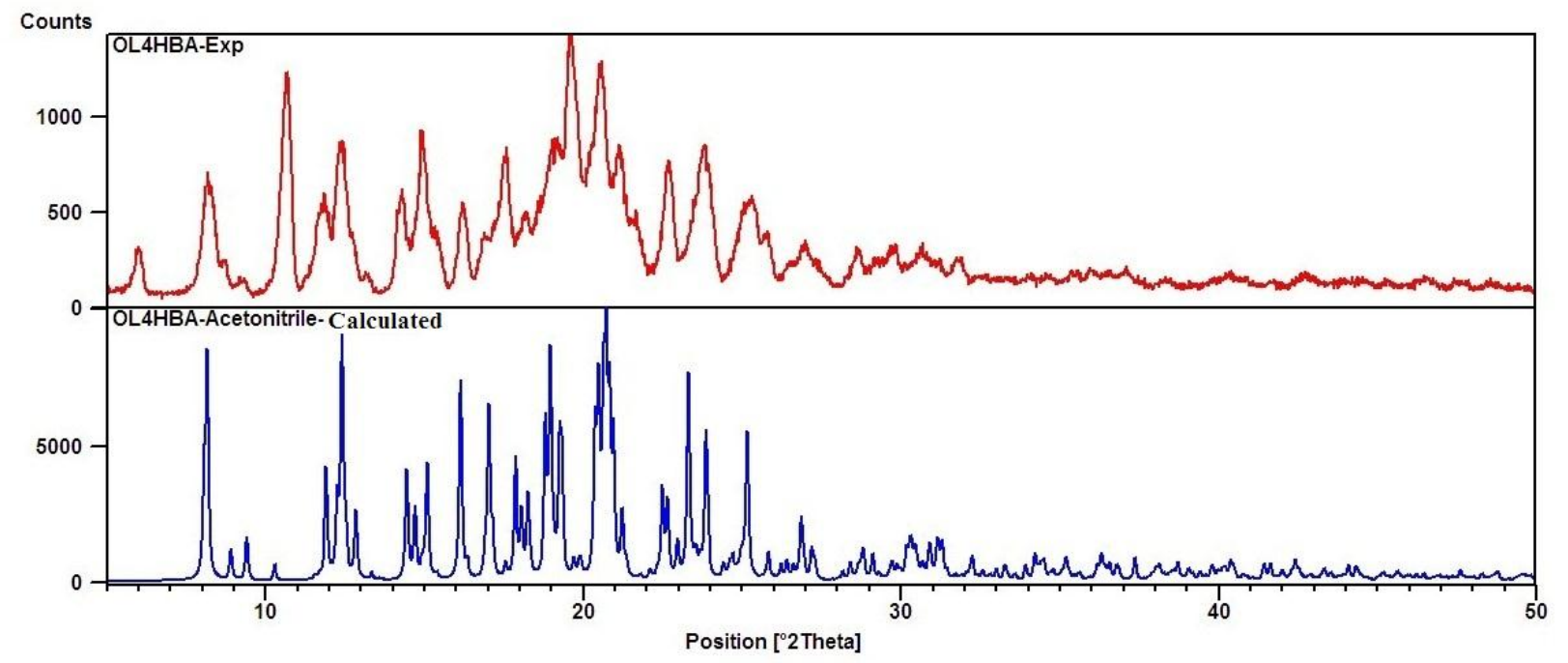

(f) Experimental (red) vs. calculated (blue) PXRD pattern of OLN•4HBA salt.

Figure S7. Comparison of PXRD patterns of all the olanzapinium salts prepared using LAG.

\section{Spectroscopic analysis}

FT-IR spectra of all the powdered samples of olanzapinium salts prepared using LAG were carried out in a Bruker instrument. The formation of olanzapinium salts can be inferred by the appearance of peak at $1400 \mathrm{~cm}^{-1}$ (symmetric $\mathrm{C}-\mathrm{O}$ streching) due to the presence of carboxylate group of salt formers. Moreover the disappeance of asymmetric stretching vibration at or above $1700 \mathrm{~cm}^{-1}$ (asymmetric $\mathrm{C}=\mathrm{O}$ stretching) is also an evidence of formation of olanzapinium salt with carboxylic acid. FT-IR spectra of all the olanzapinium salts are shown in figure S8. 


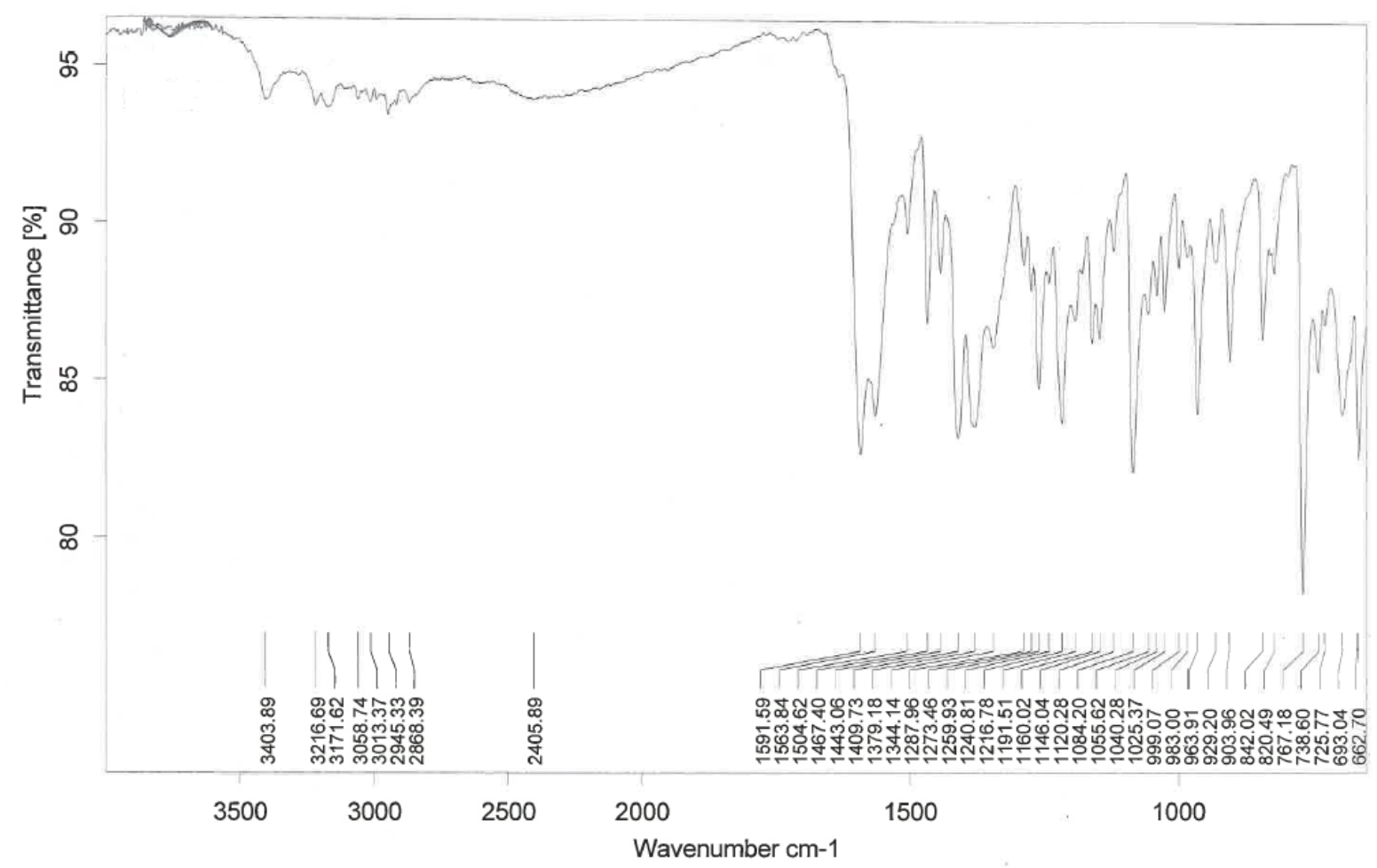

(a) Olanzapinium glycolate $(\mathrm{OLN} \cdot \mathrm{Gly})$ salt

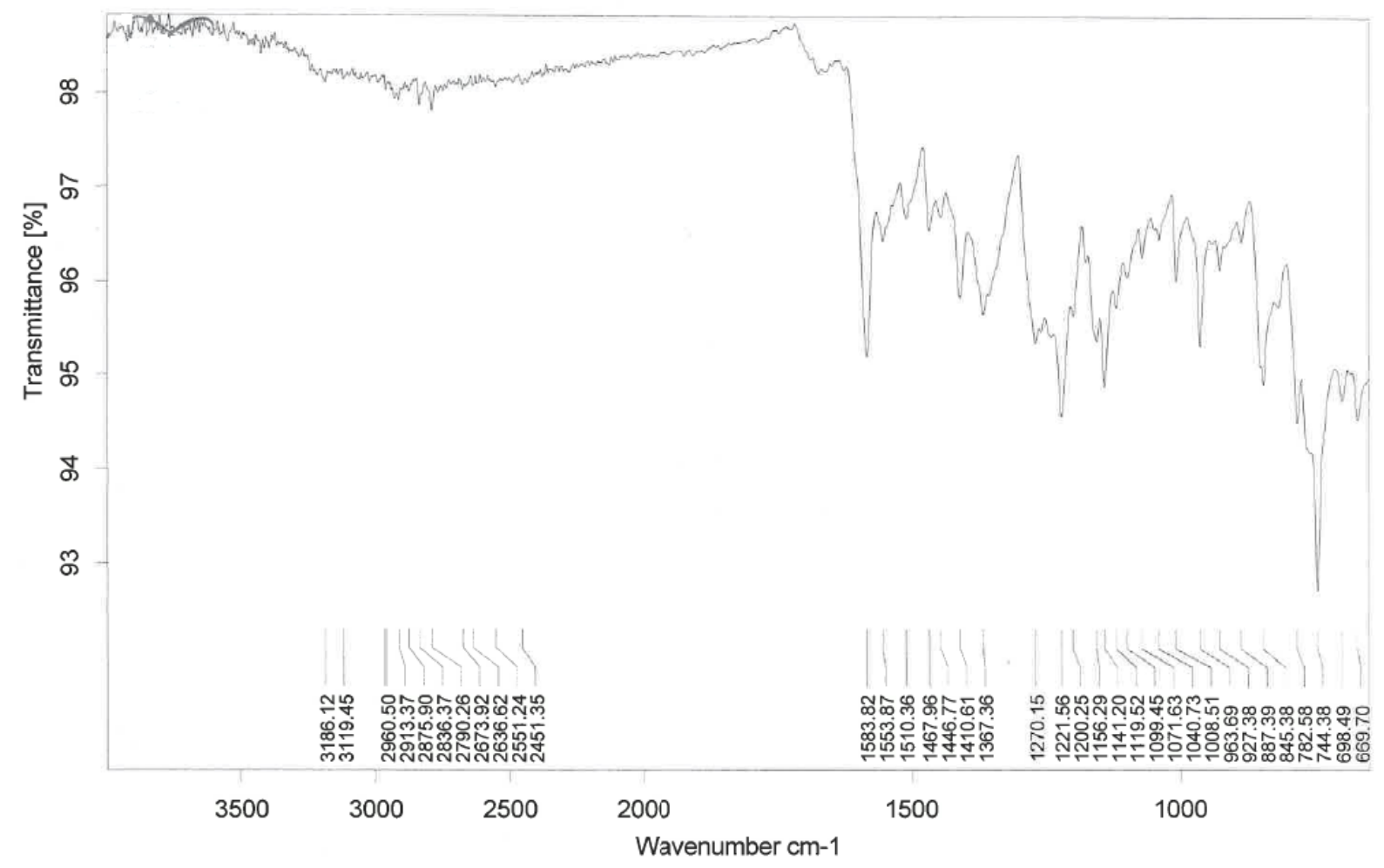

(b) Olanzapinium 4-hydroxybenzoate $(\mathrm{OLN} \bullet 4 \mathrm{HBA})$ salt 


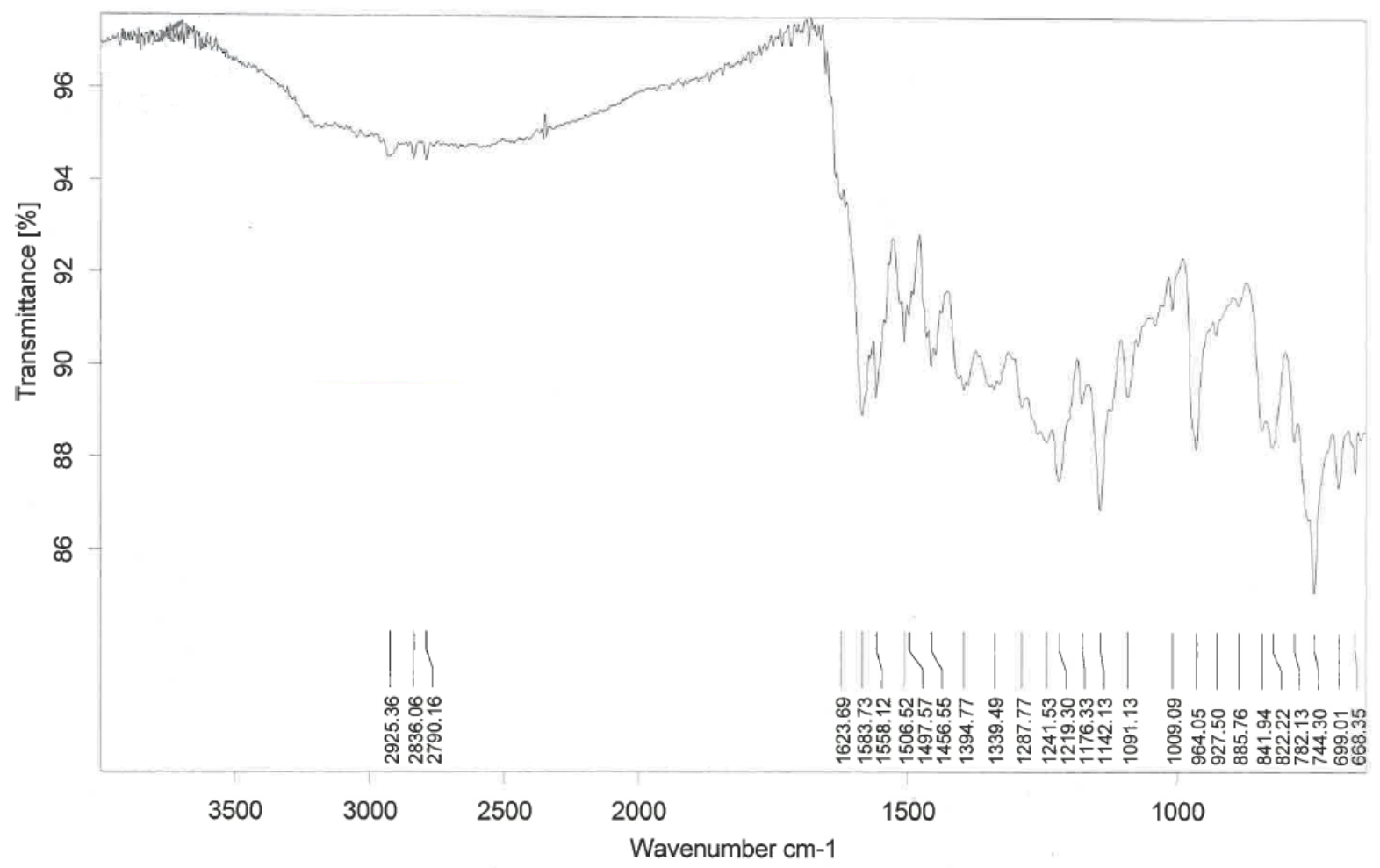

(c) Olanzapinium 2,4-dihydroxybenzoate (OLN•24DHBA) salt

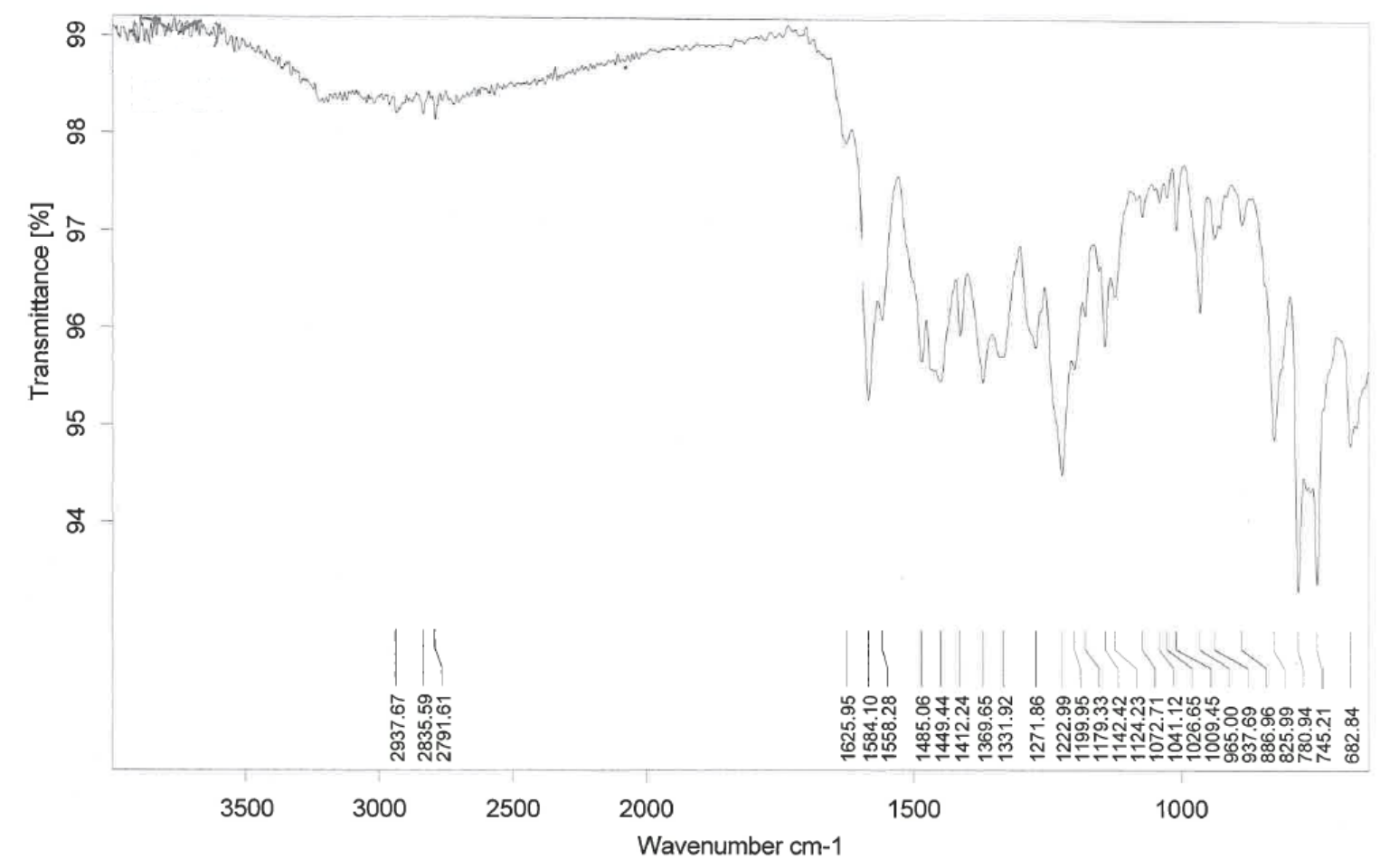

(d) Olanzapinium 2,5-dihydroxybenzoate (OLN•25DHBA) salt 


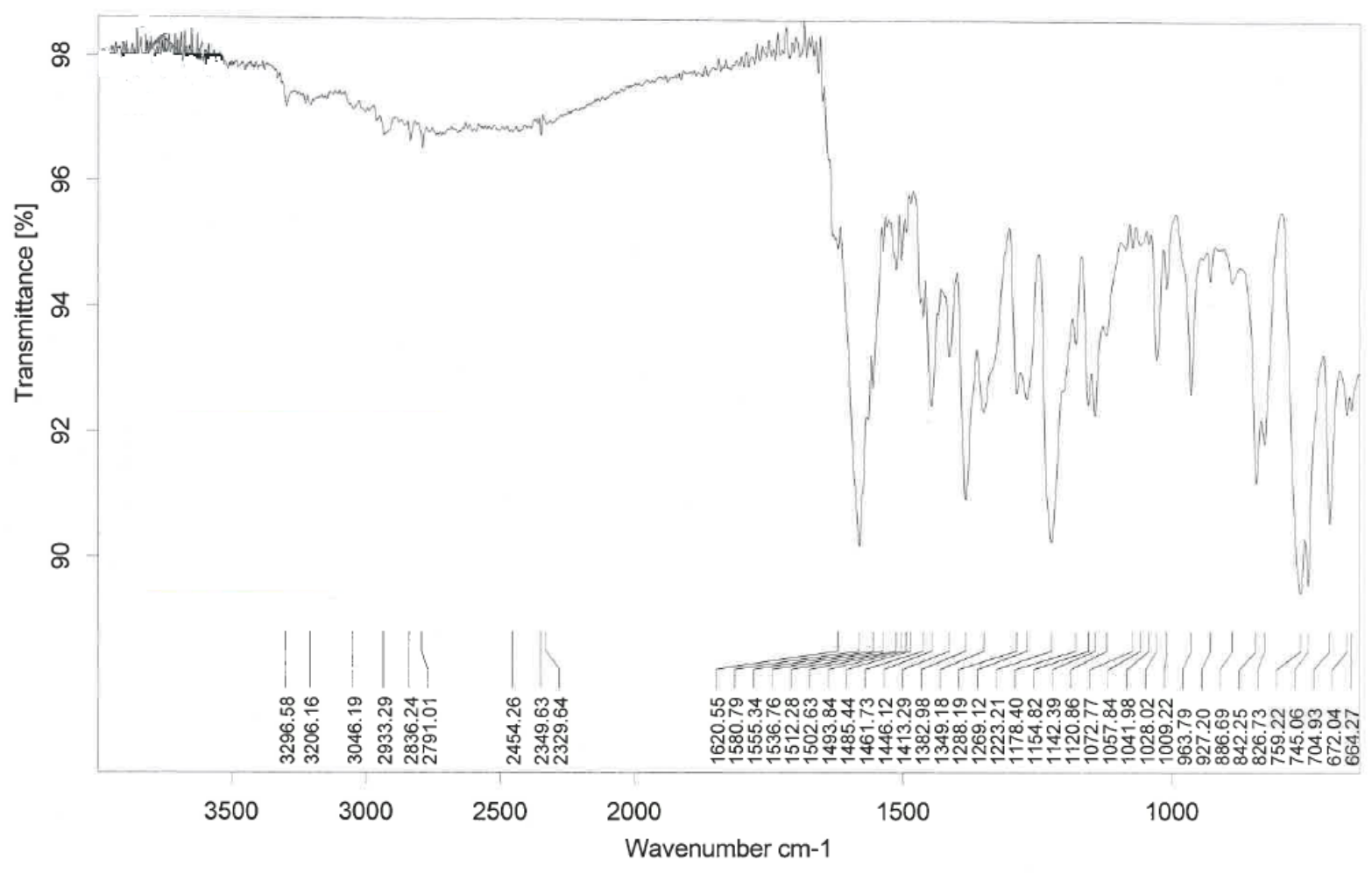

(e) Olanzapinium 2,6-dihydroxybenzoate (OLN•26DHBA) salt

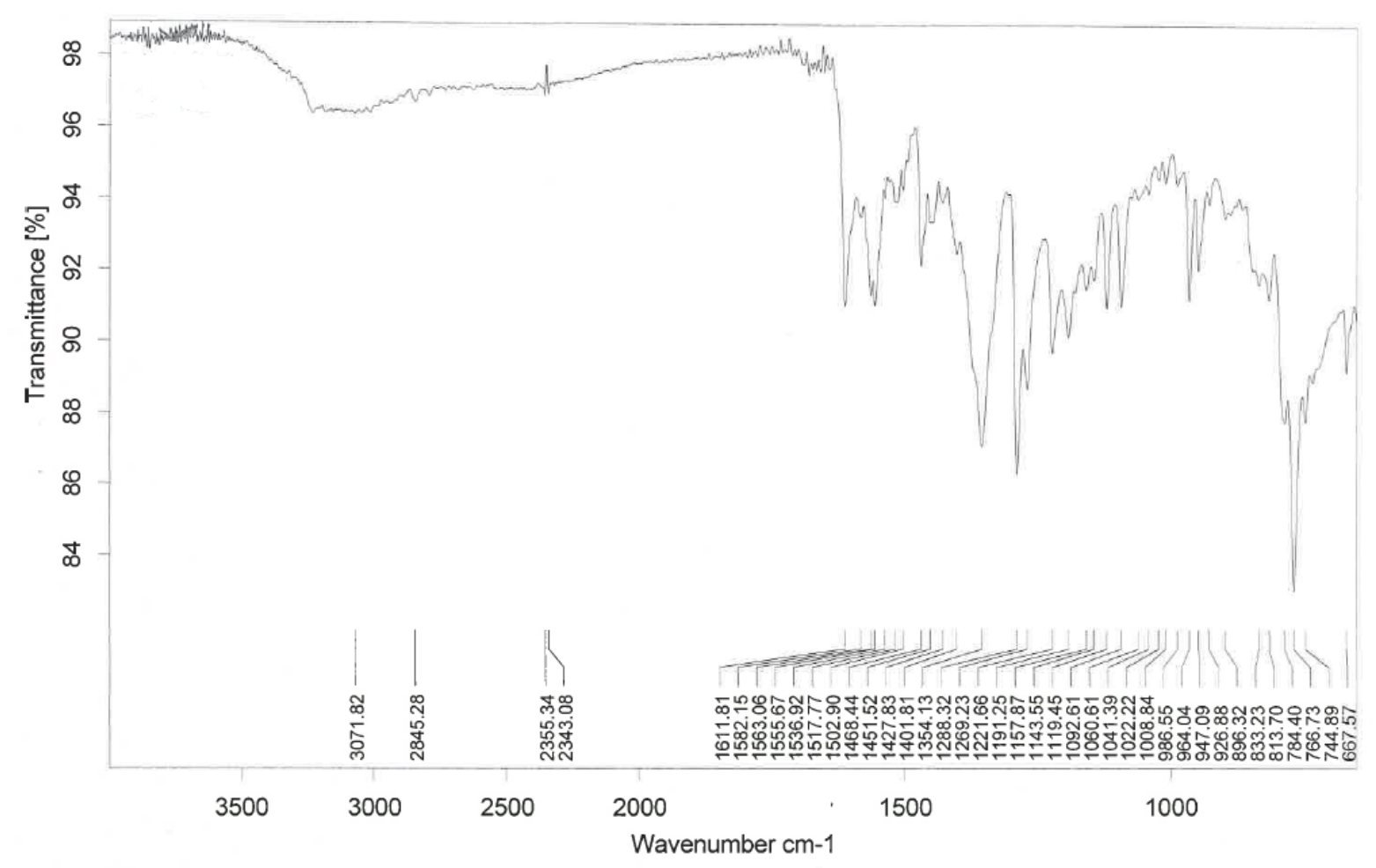

(f) Olanzapinium 3,4-dihydroxybenzoate (OLN•34DHBA) salt 


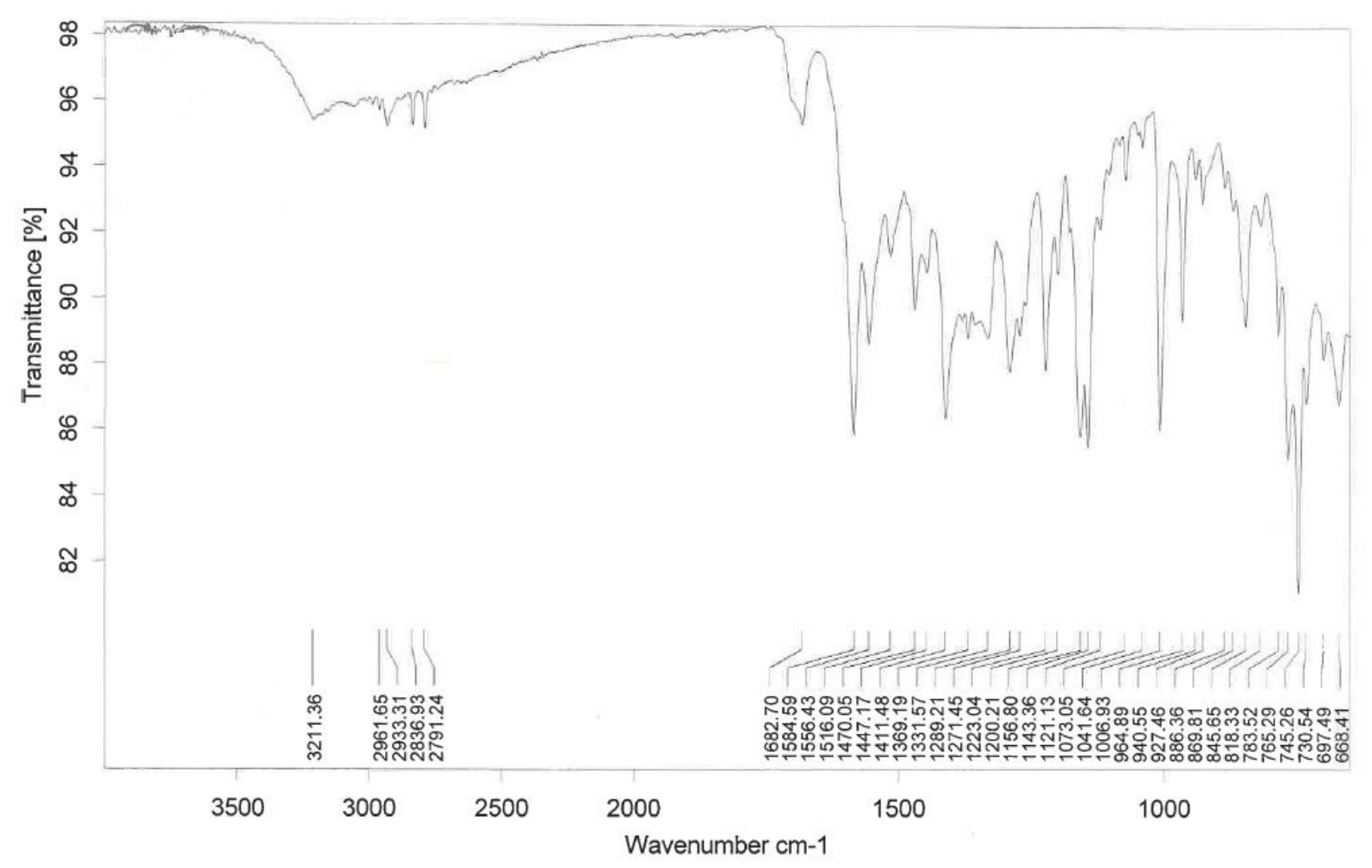

(g) Olanzapinium 3,5-dihydroxybenzoate $(\mathrm{OLN} \bullet 35 \mathrm{DHBA})$ salt

Figure S8. FT-IR spectra of all olanzapinium salts prepared using LAG (acetonitrile as liquid). Spectral region was considered from $4000-650 \mathrm{~cm}^{-1}$.

\section{Thermal analysis}

\section{Differential Scanning Calorimetry (DSC)}

Differential scanning calorimetry (DSC) of all the powdered samples of olanzapinium salts were carried out by holding the sample for $1 \mathrm{~min}$ at $30^{\circ} \mathrm{C}$ followed by heating upto $300^{\circ} \mathrm{C}$ at a heating rate of $10^{\circ} \mathrm{C} / \mathrm{min}$. All the olanzapinium salts show higher melting endotherm than the individual components except 24DHBA salt (figure S9). This anomaly may be due to poor crystal packing (lower density value) compared to other DHBA salt structure. The small exothermic peaks appeared in all powdered samples (except $\mathrm{OLN} \bullet$ Gly salt) due to crystallization of the material prior to melting event. The melting onset of all the olanzapinium salts w.r.t the salt former are listed in table S2 given below. 


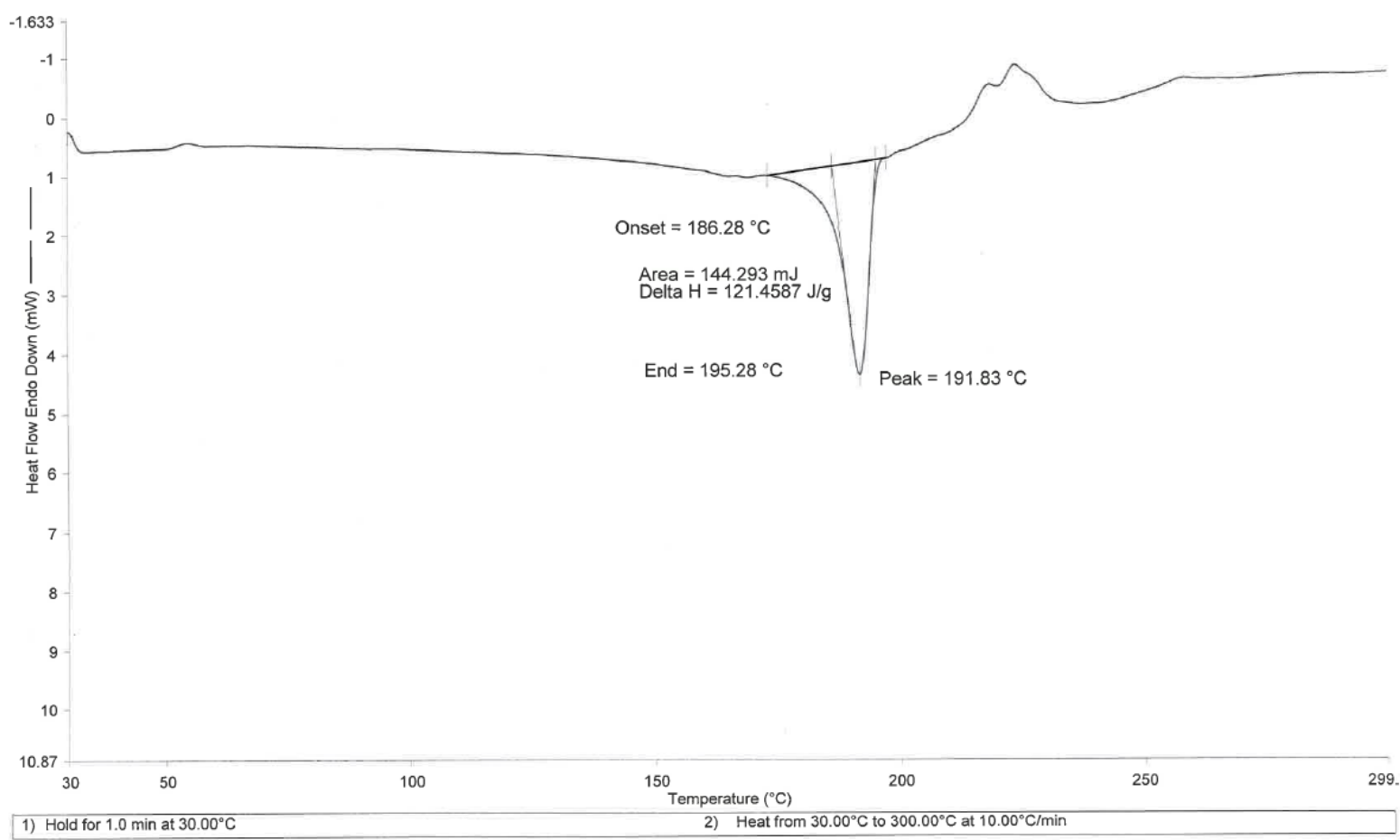

(a) Olanzapinium glycolate (OLN•Gly) salt

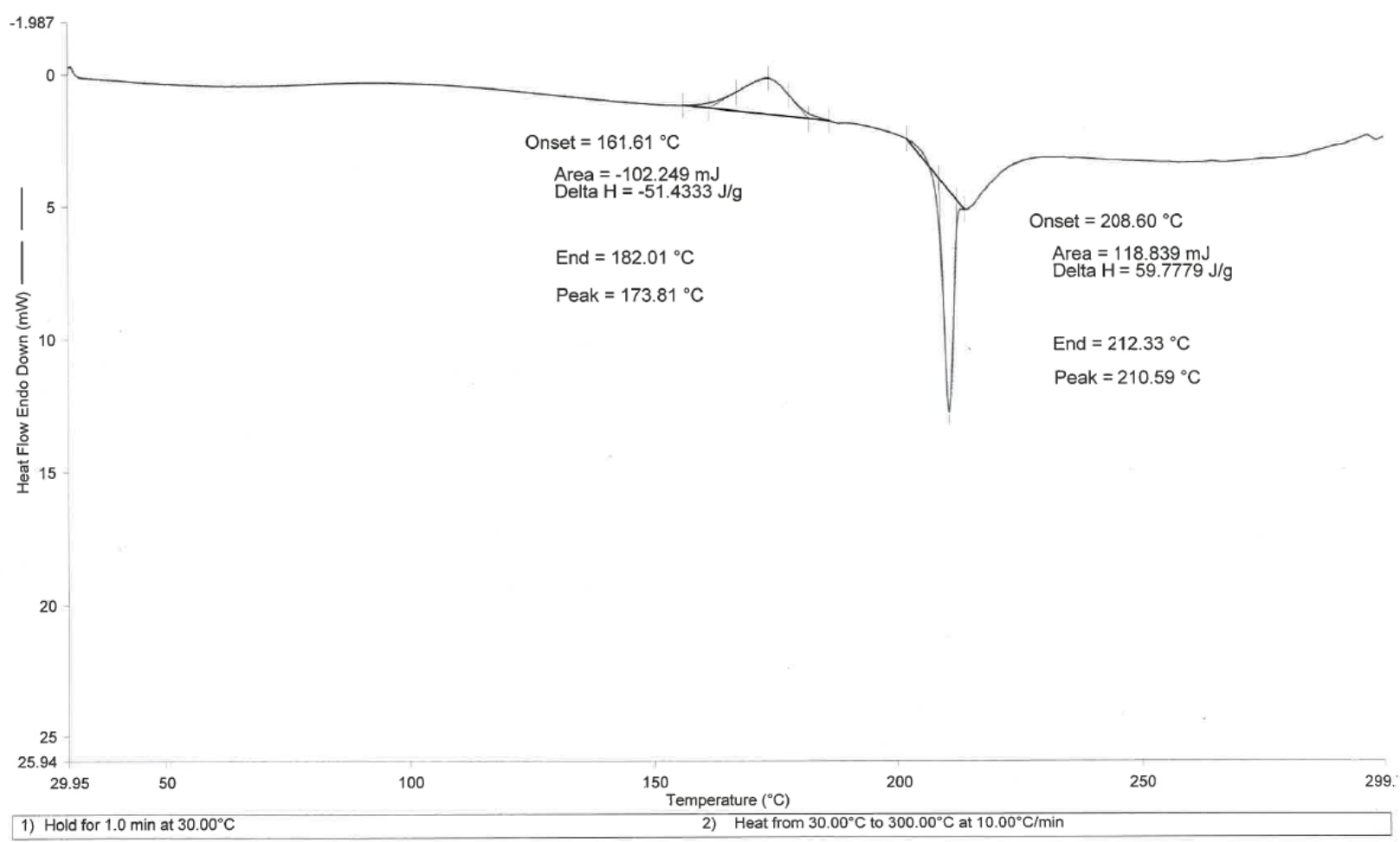

(b) Olanzapinium 4-hydroxybenzoate (OLN•4HBA) salt 


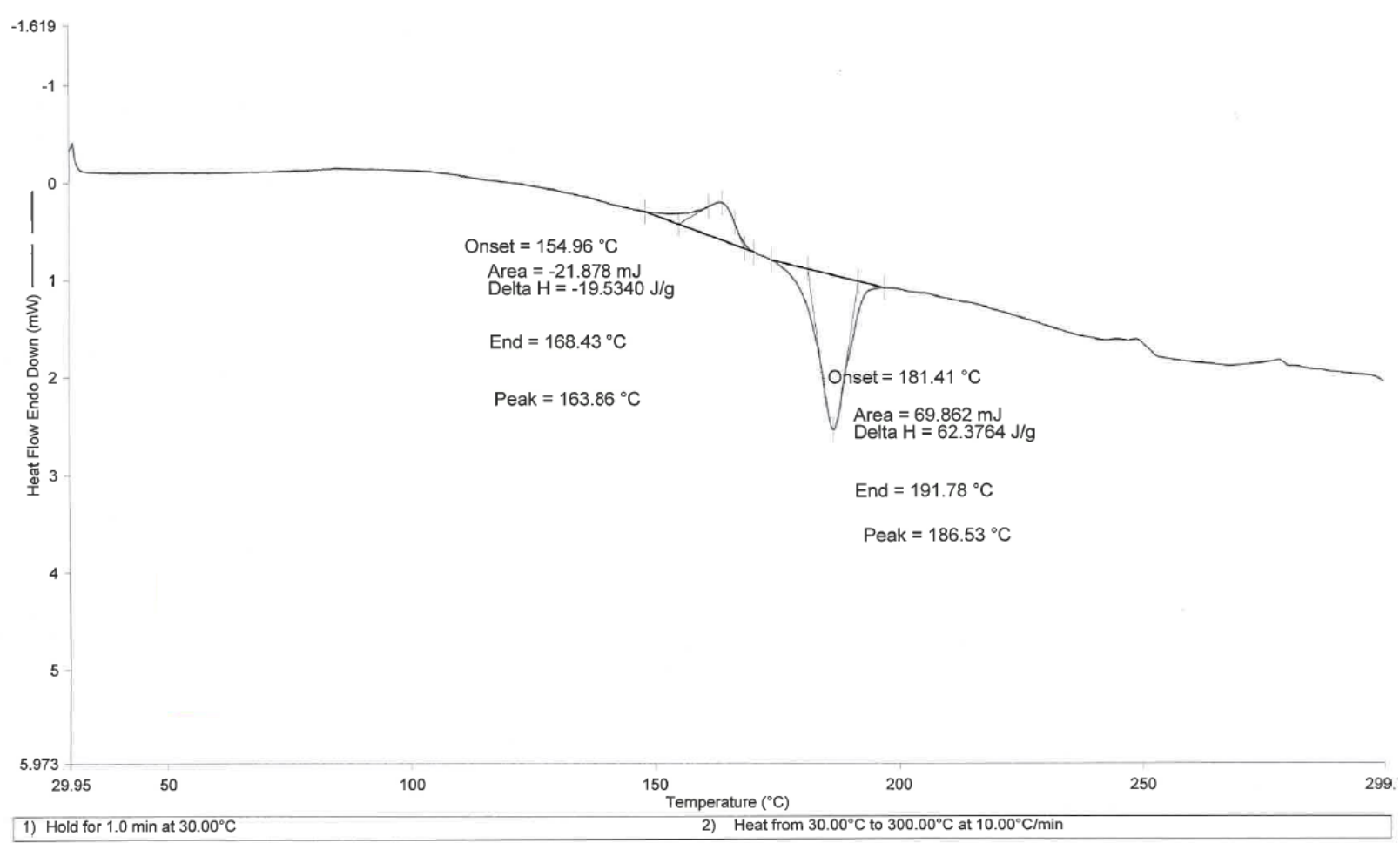

(c) Olanzapinium 2,4-dihydroxybenzoate $(\mathrm{OLN} \cdot 24 \mathrm{DHBA})$ salt

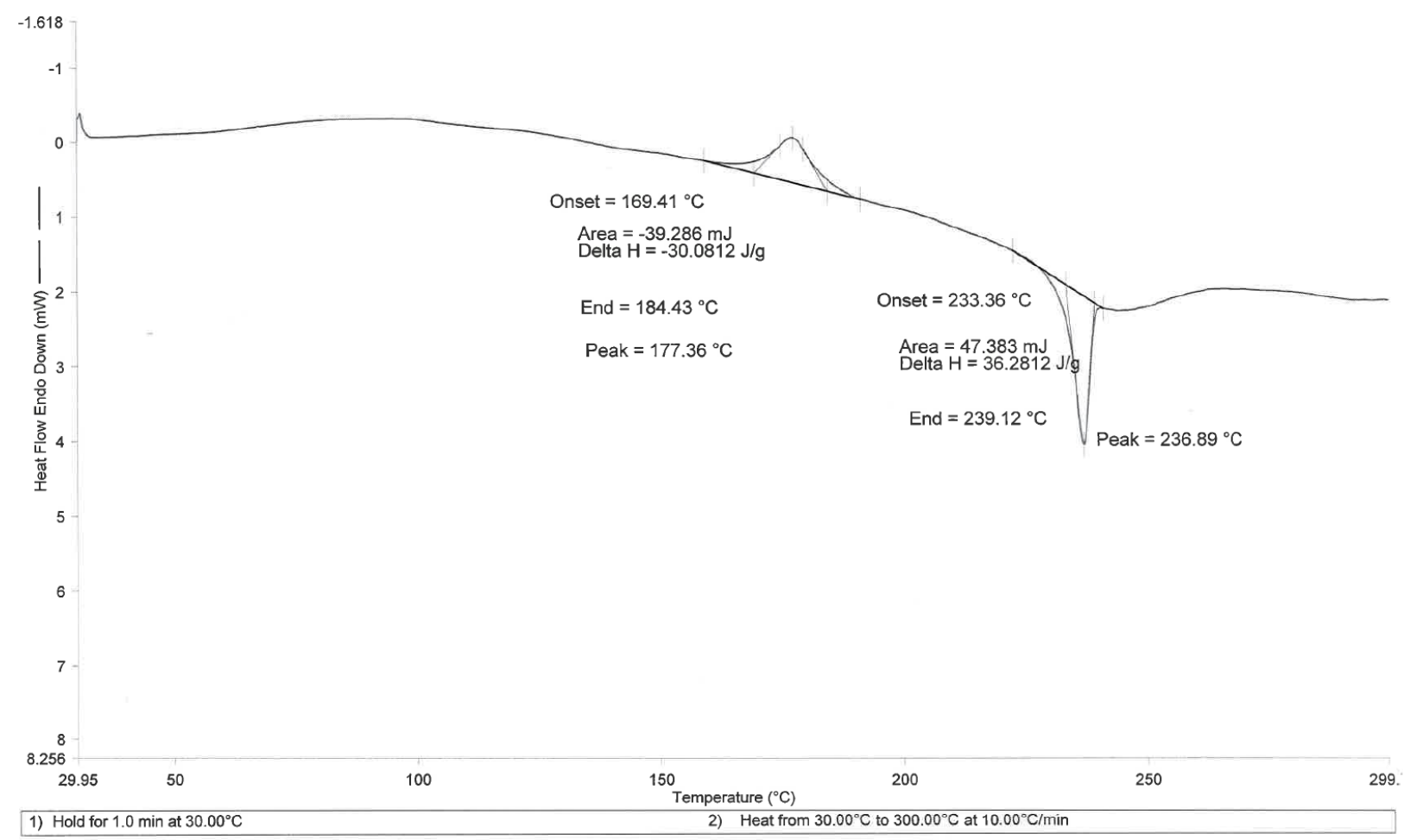

(d) Olanzapinium 2,5-dihydroxybenzoate $(\mathrm{OLN} \cdot 25 \mathrm{DHBA})$ salt 


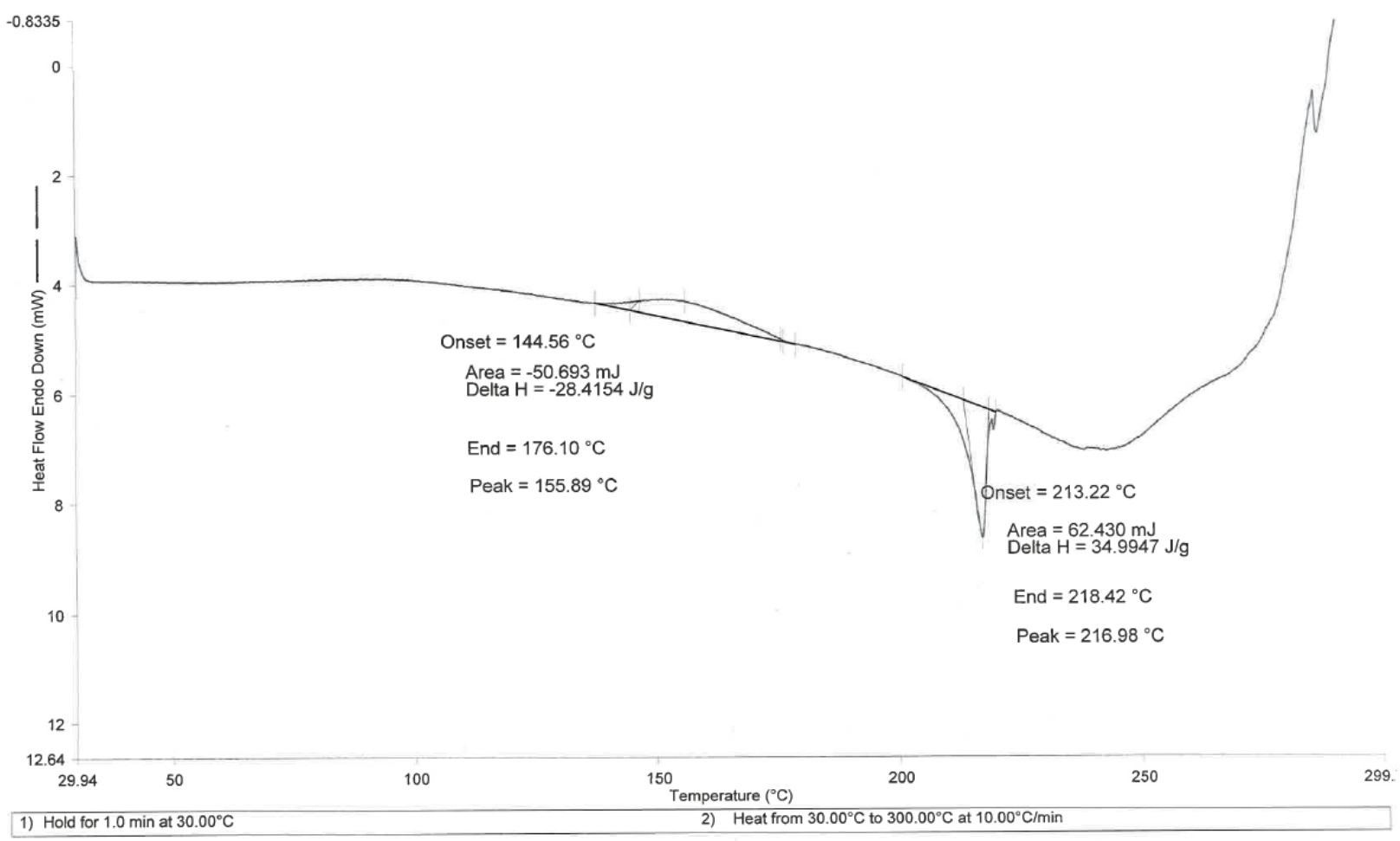

(e) Olanzapinium 2,6-dihydroxybenzoate (OLN•26DHBA) salt

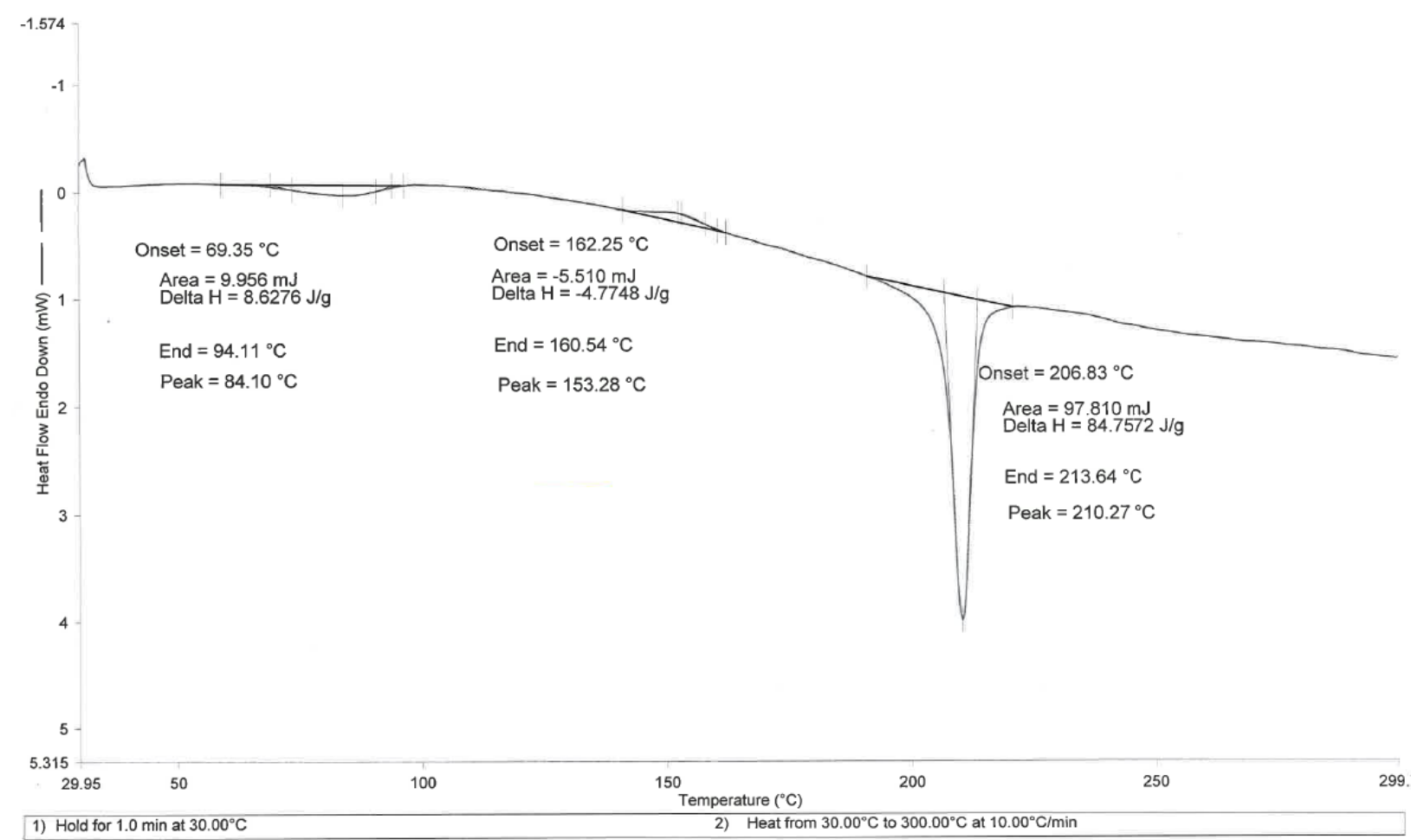

(f) Olanzapinium 3,4-dihydroxybenzoate (OLN•34DHBA) salt 


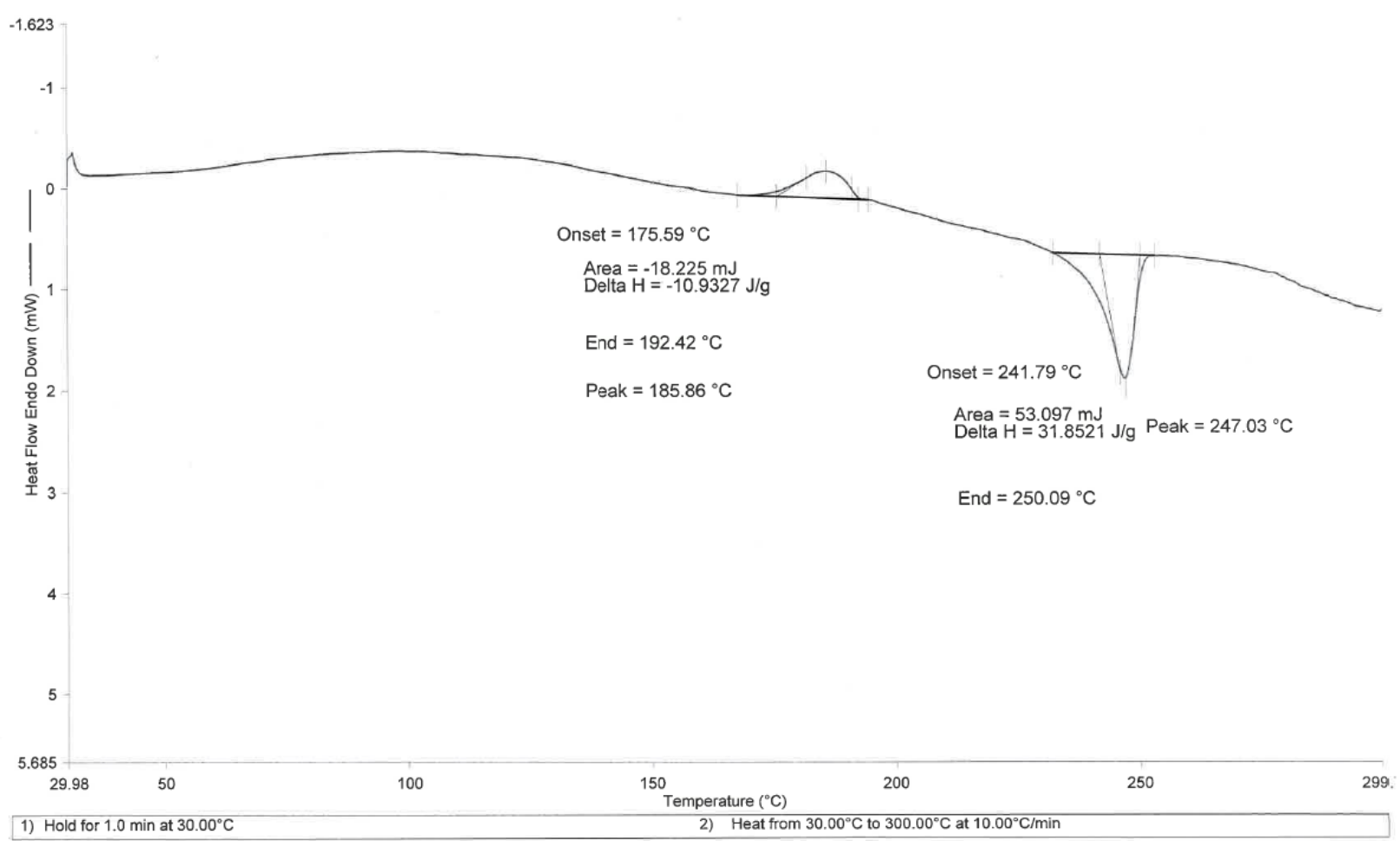

(g) Olanzapinium 3,5-dihydroxybenzoate $(\mathrm{OLN} \cdot 35 \mathrm{DHBA})$ salt

Figure S9. DSC of all the powdered olanzapinium salts prepared using LAG (acetonitrile as liquid)

Table S2. Melting points of olanzapine base, ${ }^{1}$ its salts (from DSC) and corresponding salt formers (from literature).

\begin{tabular}{|l|l|l|l|}
\hline Salt former & $\begin{array}{l}\text { Melting point }\left({ }^{\circ} \mathrm{C}\right) \\
(\text { literature })\end{array}$ & OLN salts & $\begin{array}{l}\text { Melting point }\left({ }^{\circ} \mathrm{C}\right) \\
(\text { from DSC })\end{array}$ \\
\hline & & OLN form II & 189 \\
\hline Gly & $75-80$ & OLN $\cdot G l y$ & 186 \\
\hline 4HBA & $213-217$ & OLN•4HBA & 208 \\
\hline 24DHBA & $208-211$ & OLN•24DHBA & 181 \\
\hline 25DHBA & $204-208$ & OLN•25DHBA & 233 \\
\hline 26DHBA & 165 & OLN•26DHBA & 213 \\
\hline 34DHBA & $197-200$ & OLN•34DHBA & 206 \\
\hline 35DHBA & $236-238$ & OLN•35DHBA & 241 \\
\hline
\end{tabular}




\section{Thermo-gravimetric Analysis (TGA)}

TGA experiments were performed on Perkin Elmer instrument by heating the powdered material from 30 to $300{ }^{\circ} \mathrm{C}$ at a heating rate of $20{ }^{\circ} \mathrm{C} / \mathrm{min}$. All the powdered olanzapinium salts were prepared using LAG (acetonitrile as liquid) shows a small weight loss within $100{ }^{\circ} \mathrm{C}$ which may be due to the presence of atmospheric moisture in the powder sample during analysis. The large weight loss at higher temperature corresponds to decomposition of material after melting. The TGA curves of all the samples were shown in figure S10 given below.

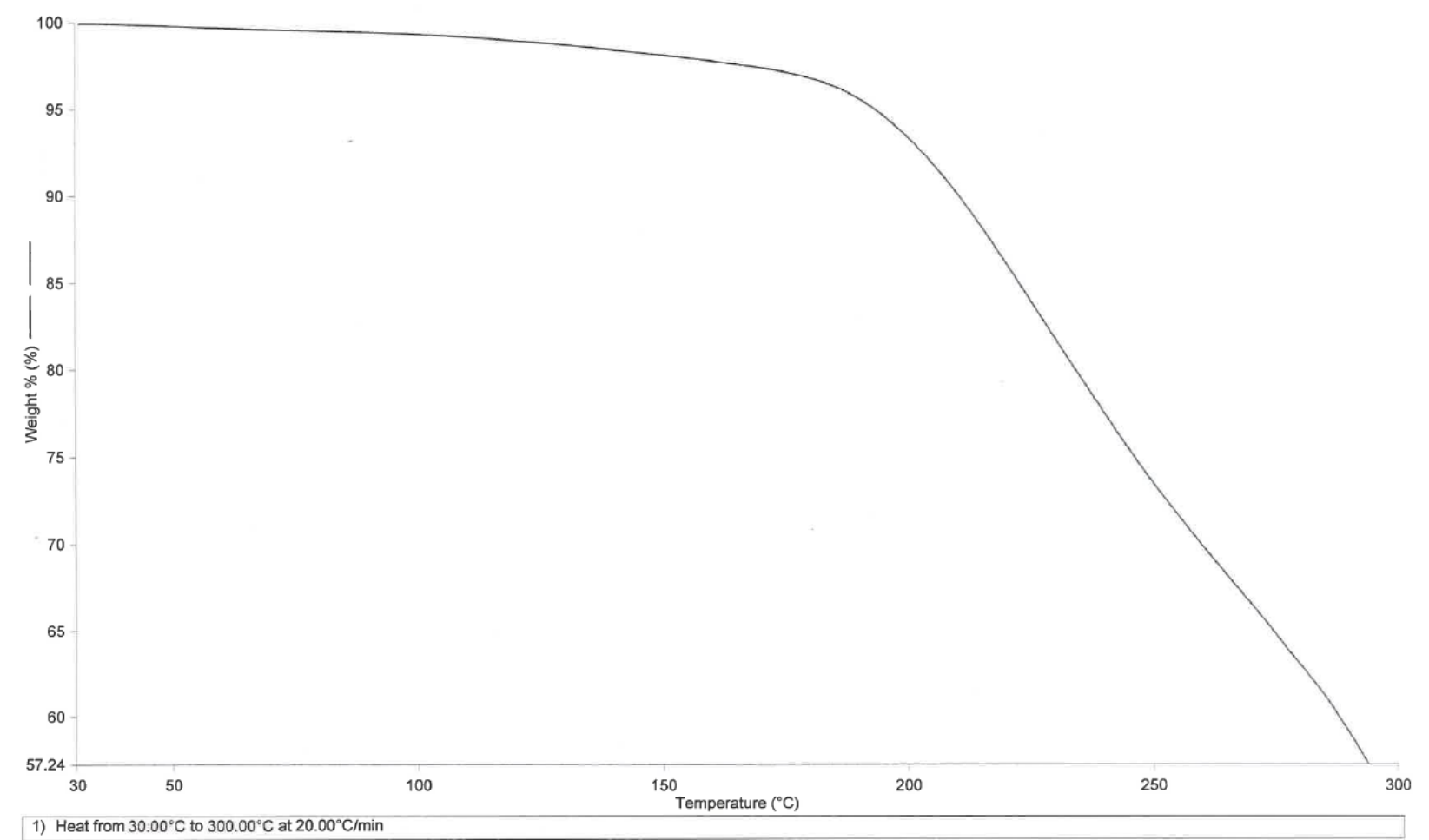

(a) Olanzapinium glycolate $(\mathrm{OLN} \cdot$ Gly) salt 


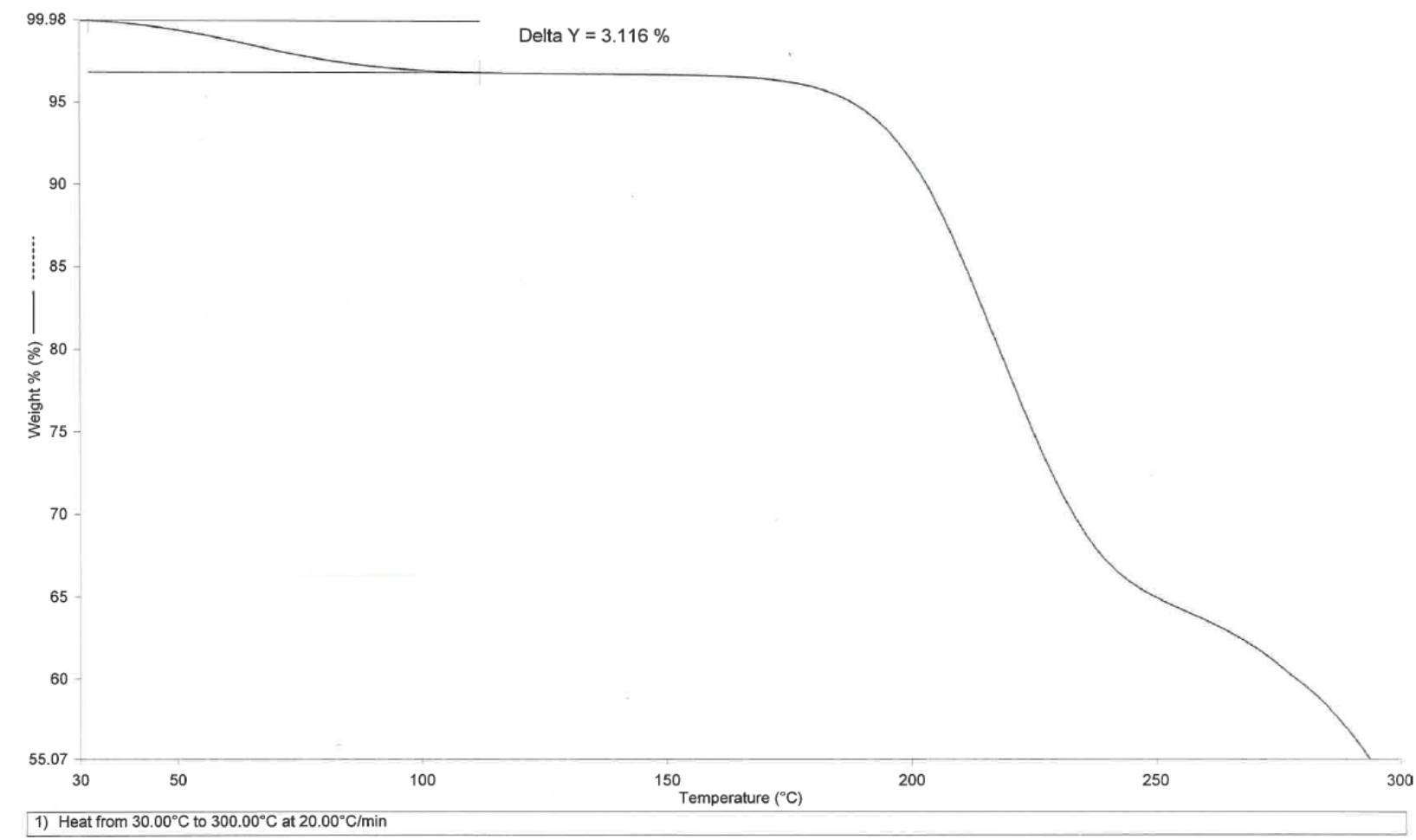

(b) Olanzapinium 4-hydroxybenzoate $(\mathrm{OLN} \bullet 4 \mathrm{HBA})$ salt

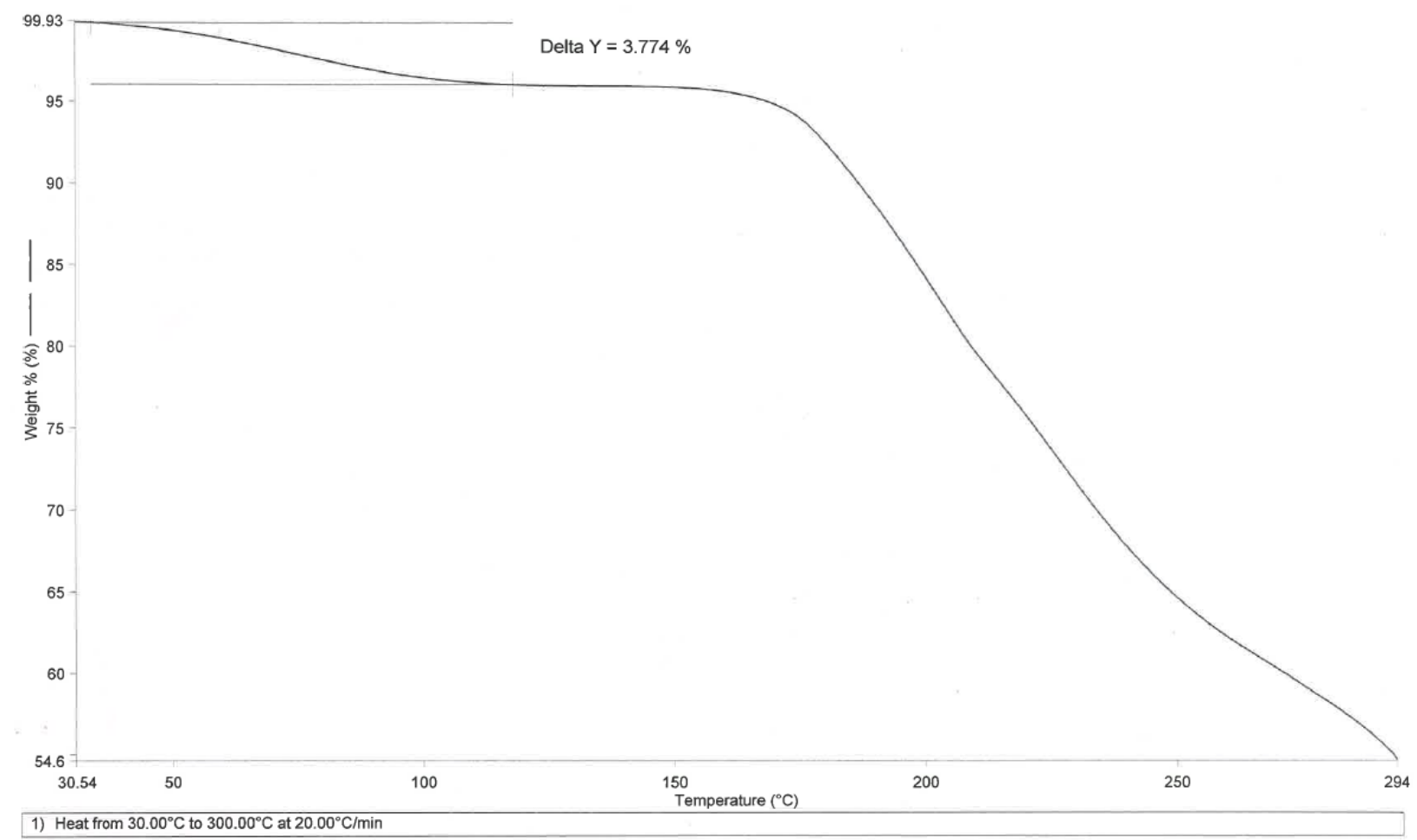

(c) Olanzapinium 2,4-dihydroxybenzoate (OLN•24DHBA) salt 


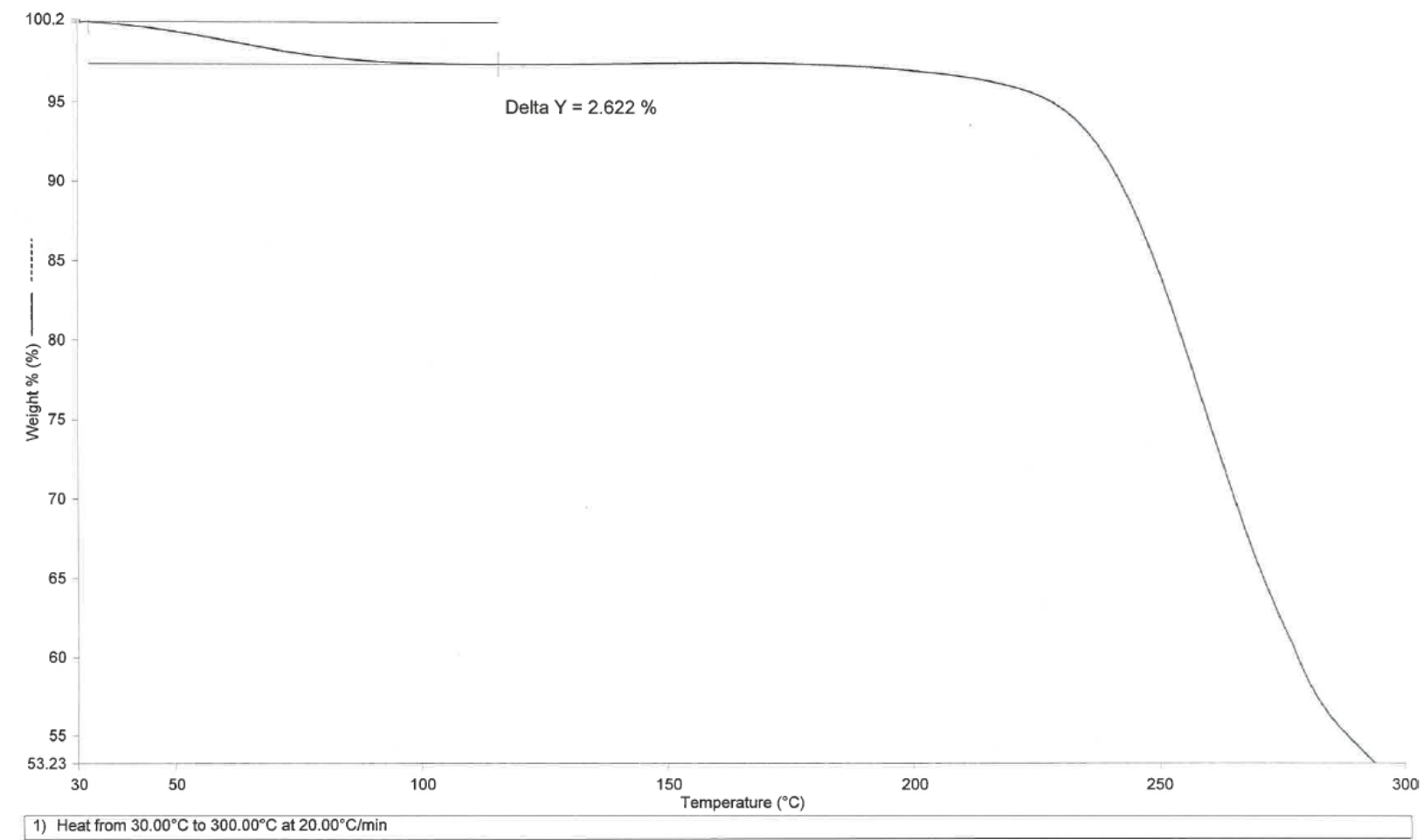

(d) Olanzapinium 2,5-dihydroxybenzoate (OLN•25DHBA) salt

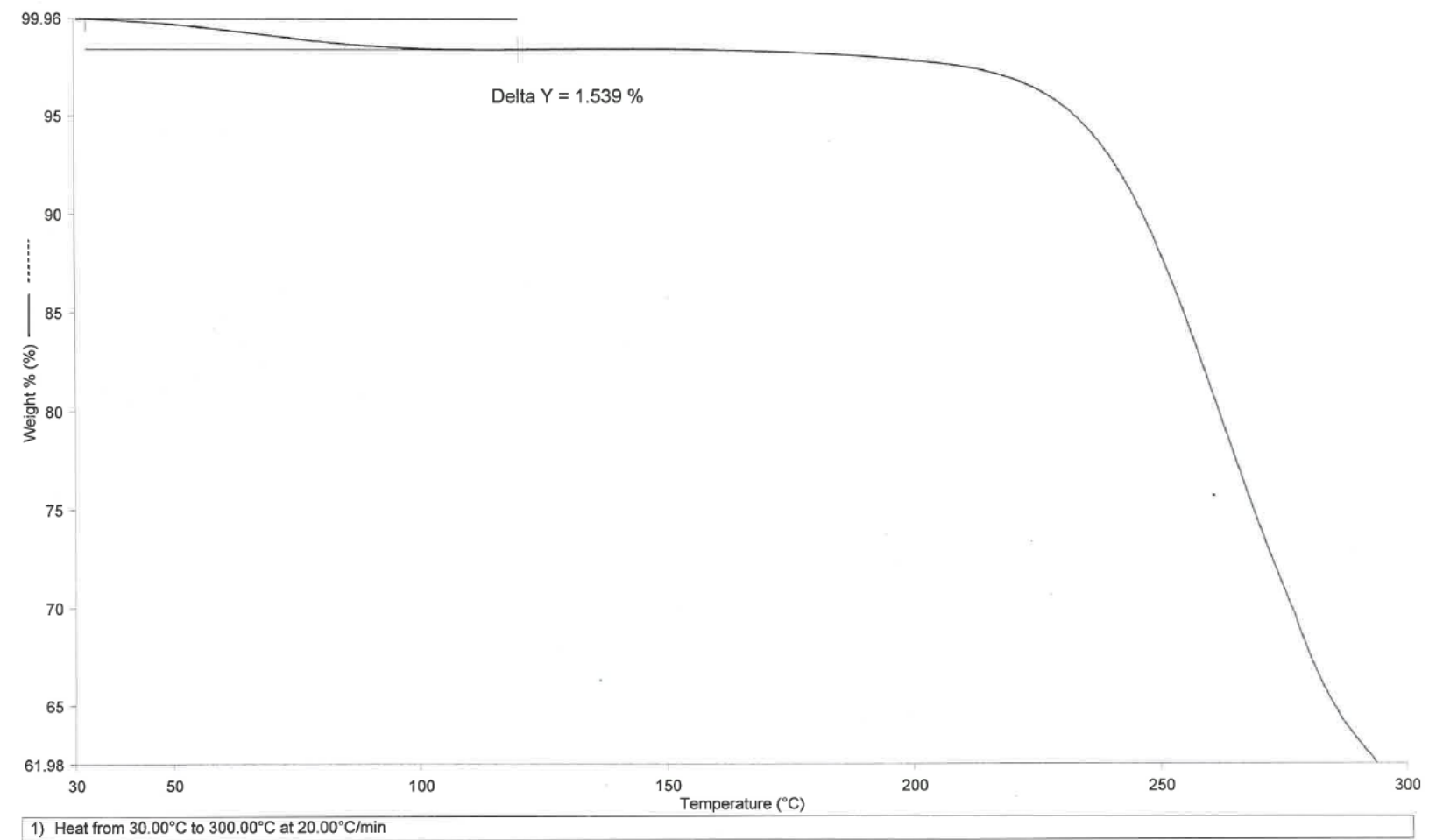

(e) Olanzapinium 2,6-dihydroxybenzoate $(\mathrm{OLN} \cdot 26 \mathrm{DHBA})$ salt 


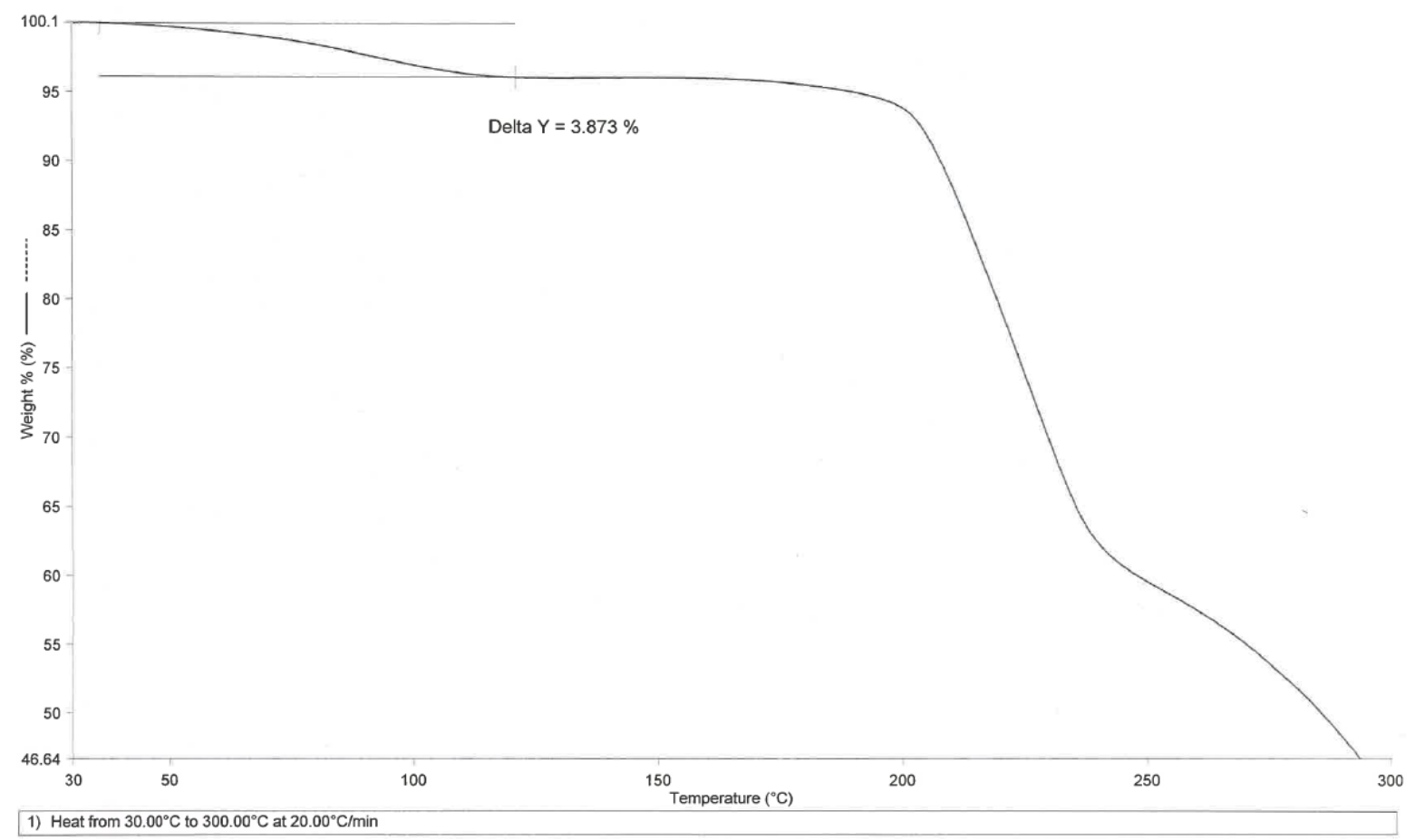

(f) Olanzapinium 3,4-dihydroxybenzoate $(\mathrm{OLN} \cdot 34 \mathrm{DHBA})$ salt

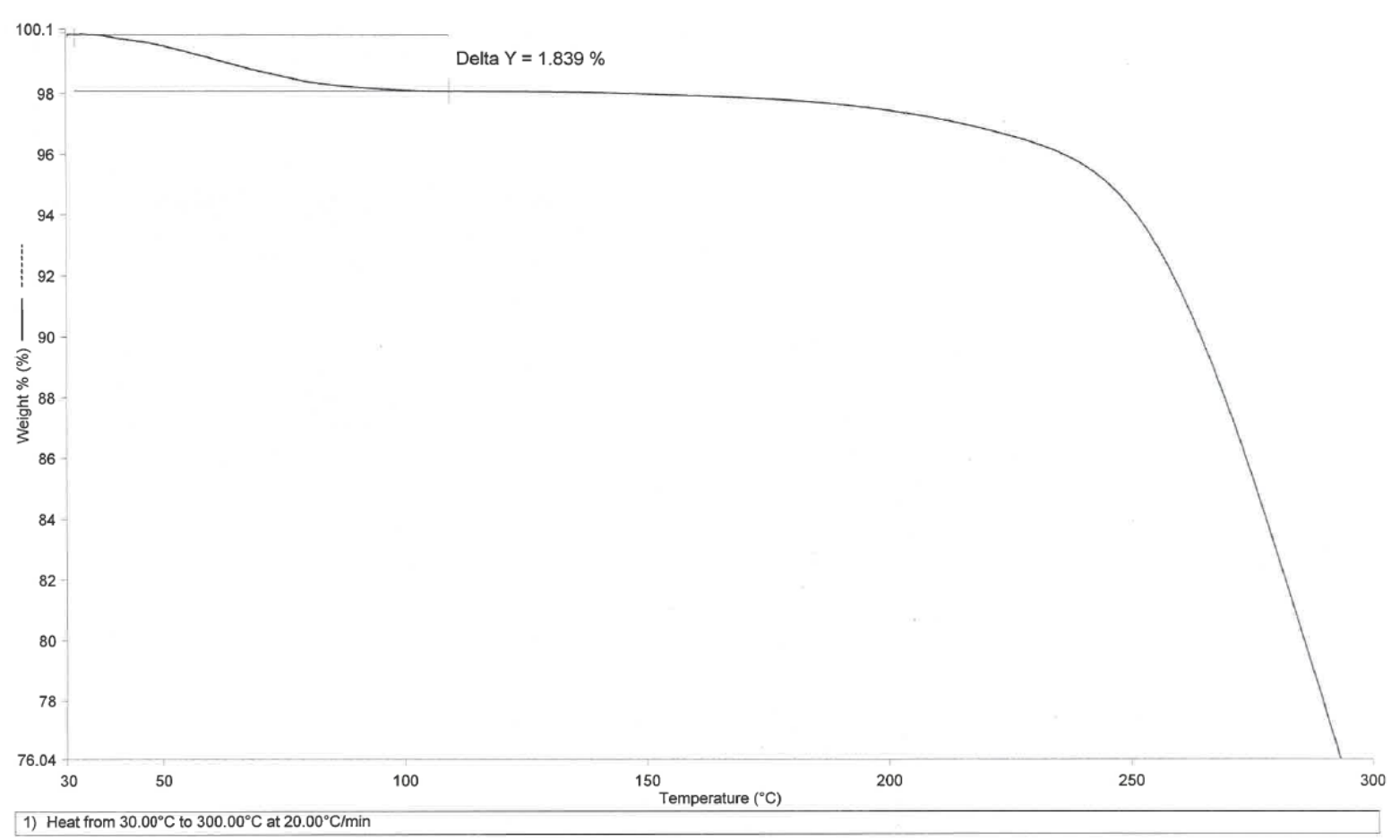

(g) Olanzapinium 3,5-dihydroxybenzoate $(\mathrm{OLN} \cdot 35 \mathrm{DHBA})$ salt

Figure S10. TGA of all powdered olanzapinium salts prepared using LAG (acetonitrile as liquid) 


\section{Reference:}

1. Thakuria, R.; Nangia, A. Cryst. Growth Des. 2013, 13, 3672-3680. 